\title{
Autoionization Dynamics of (2P1/2)ns/d States in Krypton Probed by Noncollinear Wave Mixing with Attosecond Extreme Ultraviolet and Few-Cycle Near Infrared Pulses
}

Ashley Fidler, Hugo Marroux, Erika Warrick, Etienne Bloch, Wei Cao, Stephen Leone, Daniel Neumark Submitted date: 10/06/2019 Posted date: 11/06/2019

Licence: CC BY-NC-ND 4.0

Citation information: Fidler, Ashley; Marroux, Hugo; Warrick, Erika; Bloch, Etienne; Cao, Wei; Leone, Stephen; et al. (2019): Autoionization Dynamics of (2P1/2)ns/d States in Krypton Probed by Noncollinear Wave Mixing with Attosecond Extreme Ultraviolet and Few-Cycle Near Infrared Pulses. ChemRxiv. Preprint.

The autoionization dynamics of the $\left({ }^{2} \mathrm{P}_{1 / 2}\right)$ ns/d Rydberg states in krypton are investigated using wave-mixing signals generated with subfemtosecond XUV pulses and noncollinear, few-cycle NIR pulses. Despite quantum beat oscillations from the XUV-induced coherence, these wave-mixing spectra allow for the simultaneous evaluation of autoionization lifetimes from a series of Rydberg states. Experimentally measured lifetimes for the wave-mixing signals emitting from the $\left({ }^{2} P_{1 / 2}\right) 6 d / 8 s, 7 d / 9 s$, and $8 d / 10$ s resonances compare favorably with lifetimes for the $\left({ }^{2} \mathrm{P}_{1 / 2}\right) 6 \mathrm{~d}, 7 \mathrm{~d}$, and $8 \mathrm{~d}$ Rydberg states determined from spectral linewidths. Analysis of the quantum beats reveals that the enhancement of wave-mixing pathways leads to reporter state-dependent decays in the wave-mixing signals. The results demonstrate the promise of wave-mixing spectroscopies with subfemtosecond XUV pulses to provide valuable insights into processes governed by electronic dynamics.

File list (3)

Fidler-Krypton-Autoionizing-States-Manuscript.docx (10.74 MiB) view on ChemRxiv • download file view on ChemRxiv - download file 


\title{
Autoionization Dynamics of $\left({ }^{2} \mathbf{P}_{1 / 2}\right) n s / d$ States in Krypton Probed by Noncollinear Wave Mixing with Attosecond Extreme Ultraviolet and Few-Cycle Near Infrared Pulses
}

\author{
Ashley P. Fidler ${ }^{1,2}$, Hugo J. B. Marroux ${ }^{1,2}$, Erika R. Warrick ${ }^{1,2}$, Etienne Bloch ${ }^{1}$, Wei Cao ${ }^{1,2}$, \\ Stephen R. Leone, ${ }^{1,2,3}$ and Daniel M. Neumark ${ }^{1,2}$ \\ ${ }^{1}$ Chemical Sciences Division, Lawrence Berkeley National Laboratory, Berkeley, CA 94720, USA \\ ${ }^{2}$ Department of Chemistry, University of California, Berkeley, CA 94720 USA \\ ${ }^{3}$ Department of Physics, University of California, Berkeley, CA 94720 USA
}

The autoionization dynamics of the $\left({ }^{2} \mathrm{P}_{1 / 2}\right) n s / d$ Rydberg states in krypton are investigated using spatially-isolated wave-mixing signals generated with a short train of subfemtosecond XUV pulses and noncollinear, few-cycle near infrared (NIR) pulses. Despite ubiquitous quantum beat oscillations from the XUV-induced coherences within the excited-state manifold, these wavemixing spectra allow for the simultaneous evaluation of autoionization lifetimes from a series of Rydberg states above the first ionization potential. Experimentally measured lifetimes of $22 \pm$ $8 \mathrm{fs}, 33 \pm 6 \mathrm{fs}$, and $49 \pm 6$ fs for the wave-mixing signals emitting from the $\left({ }^{2} \mathrm{P}_{1 / 2}\right) 6 \mathrm{~d} / 8 \mathrm{~s}$, $\left({ }^{2} \mathrm{P}_{1 / 2}\right) 7 \mathrm{~d} / 9 \mathrm{~s}$, and $\left({ }^{2} \mathrm{P}_{1 / 2}\right) 8 \mathrm{~d} / 10$ s resonances compare favorably with lifetimes for the $\left({ }^{2} \mathrm{P}_{1 / 2}\right) 6 \mathrm{~d}, 7 \mathrm{~d}$, and $8 \mathrm{~d}$ Rydberg states determined from spectral linewidths. Analysis of the quantum beats reveals that the enhancement of wave-mixing pathways that couple the $\left({ }^{2} \mathrm{P}_{1 / 2}\right) n d$ states to themselves leads to individual reporter state-dependent decays in the wave-mixing signals. The results demonstrate the promise of wave-mixing spectroscopies with subfemtosecond XUV pulses to provide valuable insights into processes governed by electronic dynamics.

\section{Introduction}

Dynamics governed by electronic processes occur on some of the shortest timescales in nature, ranging from hundreds of attoseconds $\left(1 \mathrm{as}=10^{-18} \mathrm{~s}\right)$ to a few femtoseconds $\left(1 \mathrm{fs}=10^{-15} \mathrm{~s}\right) .^{1-3}$ Until recently, the lifetimes of these ultrafast processes typically have been determined through spectral lineshape measurements in the frequency domain. However, effects such as finite spectral resolution, heterogenous and homogeneous broadening, and spectral congestion often prevent the retrieval of accurate lifetimes associated with the underlying electronic dynamics. ${ }^{4}$ With the advent of high harmonic generation (HHG)-based techniques to produce attosecond pulses in the extreme ultraviolet (XUV) and soft x-ray regimes, these dynamics have become accessible to direct measurement in the time domain. Transient absorption and photoelectron techniques utilizing attosecond pulses have been developed and employed to measure dynamic processes including autoionization, ${ }^{4,5}$ Auger decay, ${ }^{6}$ and charge migration. ${ }^{7}$ However, the observed time-dependent spectra are often complicated by overlapping spectral effects, precluding quantitative measurements in complex systems. Here, nonlinear wave-mixing spectroscopy is utilized to investigate autoionization in krypton without the interference of many of these complicating effects.

Nonlinear spectroscopies are regularly employed in the optical, infrared, and radiofrequency regimes to probe complex systems with high selectivity. ${ }^{8,9}$ In these investigations, intense electromagnetic fields coherently induce a macroscopic polarization in a target medium with significant contributions from higher-order terms, which can be expressed as: 


$$
P=\varepsilon_{0}\left(\chi^{(1)} E+\chi^{(2)} E E+\chi^{(3)} E E E+\ldots\right)
$$

where $\varepsilon_{0}$ is the vacuum permittivity, $\chi^{(n)}$ is the $n^{\text {th }}$-order susceptibility, and $E$ is the electric field of the excitation. In an isotropic medium, these higher-order terms result in emission from nonlinear, or wave-mixing, processes dependent upon odd orders of $x^{(n)}$. Nonlinear techniques capitalize on the conservation of momentum and energy in the generation of the newly emitted field to resolve and measure signals that are often obscured in other techniques. Examples include measurements of energy transfer dynamics in light harvesting complexes with two-dimensional electronic spectroscopy, ${ }^{10-12}$ studies probing the properties of surfaces and interfaces with second harmonic generation (SHG)-based methods, ${ }^{13,14}$ and imaging in biological samples with coherent anti-stokes Raman microscopy. ${ }^{15,16}$

In our laboratory, we have developed a nonlinear XUV spectroscopy utilizing attosecond XUV pulses produced by HHG and two few-cycle NIR pulses to probe electronic dynamics in atoms and small molecules. The implementation of a noncollinear beam geometry between the three pulses has proven particularly powerful, as it facilitates the spatial separation of desired wavemixing signals through phase-matching. These experiments have successfully measured both electronic and vibrational wave packet dynamics, ${ }^{17-21}$ characterized the outer well of a previously inaccessible dark state in $\mathrm{N}_{2},{ }^{20}$ and produced the first experimental demonstration of two dimensional spectroscopy in the XUV. ${ }^{21}$ In a recent study, the nonperturbative nature of noncollinear wave-mixing with these ultrashort, intense pulses was explored in He, revealing that the accumulation of the AC Stark phase over the NIR pulse duration leads to delays in higherorder signal generation. ${ }^{22}$ However, all of these experiments were performed on systems containing only long-lived Rydberg states, and the generation and time dependence of nonlinear signals from XUV-excited states that decay on ultrafast timescales has yet to be established.

In this work, multiple orders of spatially-isolated wave-mixing signals from bound and autoionizing states are generated in krypton using a noncollinear geometry between a short train of subfemtosecond XUV pulses and two time-coincident few-cycle NIR pulses. The resulting wave-mixing spectra probe the autoionization dynamics of an extended manifold of Rydberg states simultaneously. Pronounced quantum beating provides detailed information about the evolving XUV-induced coherence and NIR-mediated coupling of Rydberg states. Despite extensive coupling within the manifold of states, lifetimes of individual $4 s^{2} 4 \mathrm{p}^{5}\left({ }^{2} \mathrm{P}_{1 / 2}\right) n d$ autoionizing states can be retrieved directly in the time domain, yielding values very close to those calculated from frequency-domain linewidth measurements. Fourier analysis reveals dominant contributions from wave-mixing pathways that preferentially couple the $\left({ }^{2} \mathrm{P}_{1 / 2}\right) n d$ autoionizing states with themselves, leading to the observed reporter-state specific decays. While the mechanism is still unknown, the relative enhancement of these pathways does not appear to arise from a purely perturbative picture of wave-mixing, suggesting that nonperturbative effects may impact nonlinear signal evolution in this system. These results demonstrate that wavemixing spectroscopies constitute a powerful method for the study of ultrafast dynamics in the XUV regime, providing an alternative to spectrally complex transient absorption measurements.

\section{Experimental Methods}

The apparatus utilized for background-free attosecond wave-mixing measurements has been described previously $\mathrm{y}^{20,22}$ and is shown in Fig. 1a. A $2 \mathrm{~mJ}$ per pulse, $1 \mathrm{kHz}$ repetition rate 
Ti:sapphire commercial femtosecond laser system (Femtopower HE, Femtolasers) produces 22 fs NIR pulses at a central wavelength of $760 \mathrm{~nm}$. The output spectrum is spectrally broadened in a $400 \mu \mathrm{m}$ inner diameter hollow core fiber filled with 1.2 bar of neon gas and is subsequently recompressed in time by six pairs of broadband chirped mirrors (Ultrafast Innovations) and a 1 mm thick ammonium dihydrogen phosphate (ADP) plate, ${ }^{23}$ resulting in 6 fs pulses that span a wavelength range from 550 to $950 \mathrm{~nm}$. A 50:50 beamsplitter divides the broadband light into two arms. The light transmitted through the beamsplitter is focused by a $50 \mathrm{~cm}$ focal length mirror into a vacuum chamber at $10^{-3} \mathrm{~Pa}\left(10^{-6}\right.$ Torr $)$ that contains a cell statically filled with xenon gas for high harmonic generation. The interaction of the few-cycle NIR pulse with the gas generates a pulse train consisting of $2-3$ subfemtosecond XUV pulses. A $0.1 \mu \mathrm{m}$ In foil (Lebow) attenuates the driving NIR beam and selects harmonics between 11 and $17 \mathrm{eV}$, allowing for excitation of states both below and above the first ionization potential (IP) of $\mathrm{Kr}$ at $14.0 \mathrm{eV}$. A gold-coated toroidal mirror focuses the XUV through an annular mirror into a $1 \mathrm{~mm}$ pathlength (diameter) sample cell containing $1467 \mathrm{~Pa}$ (11 Torr) of krypton gas. Holes approximately $500 \mu \mathrm{m}$ in diameter drilled through both sides of the HHG and sample cells allow for the passage of light and continuous escape of the used gas. The XUV intensity is estimated to be $10^{8}-10^{10} \mathrm{~W} \mathrm{~cm}-2$ in the target cell due to the low conversion efficiency of HHG.

The intensity necessary to support nonlinear processes is provided by the NIR light reflected by the 50:50 beamsplitter. These few-cycle NIR pulses are delayed relative to the XUV with a piezoelectric stage (P-622 with E509 controller, Physik Instrumente (PI)) and then split into two separate arms by a 50:50 beamsplitter. Two $1 \mathrm{~m}$ focal length mirrors direct the reflected and transmitted arms vertically above and below the hole of the annular mirror in the vacuum chamber before achieving beam diameters of $100 \mu \mathrm{m}$ (FWHM) and a combined intensity of

$2 \times 10^{12} \quad \mathrm{~W} \mathrm{~cm}^{-2}$ in the target cell. To ensure spatial separation of signals originating from multiphoton wave-mixing pathways from the harmonics, the two NIR beams intersect the XUV in a noncollinear geometry at angles of approximately $18 \mathrm{mrad}\left(1^{\circ}\right)$ and $13 \operatorname{mrad}\left(0.75^{\circ}\right)$ for the upper and lower arms, respectively (Fig. 1b). In the experiments described here, the two NIR pulses are time-coincident. A second piezoelectric stage is placed in the arm transmitted through the 50:50 beamsplitter to compensate for daily fluctuations in the length of the two arms. Temporal and spatial overlap between the XUV and both NIR arms is determined by removing the metallic foil blocking the copropagating NIR after HHG and inserting a removable mirror into the beam path after the annular mirror, directing all three NIR beams outside the vacuum apparatus to an optical CMOS camera (DCC1545M, Thorlabs). The positions of the stages are modified manually until interference fringes are observed between all beams (Supplementary Fig.1). The crossing angle $\theta_{\text {crossing }}$ can be calculated from the fringe spacing via Eqn. (2):

$$
\theta_{\text {crossing }}=2 \sin ^{-1}\left(\frac{\lambda_{N I R}}{2 x}\right)
$$

where $\lambda_{N I R}$ is the central NIR wavelength and $x$ is the fringe spacing.

A $0.15 \mu \mathrm{m}$ In foil (Lebow) attenuates the NIR beams after the sample cell. The transmitted XUV light is dispersed in frequency by a gold-coated flat-field grating (01-0464, Hitachi) and recorded as a function of both frequency and phase-matching divergence angle by a $1340 \times 400$ pixel $\mathrm{x}$-ray CCD camera (Pixis XO 400B, Princeton Instruments). From the absorption features of long-lived Rydberg states in argon, the spectral resolution at $14.3 \mathrm{eV}$ is expected to be $19 \mathrm{meV}$. Signal from a total of 1500 laser pulses is accumulated at each XUV-NIR delay. Here, positive 
delays indicate that the XUV pulse precedes the time coincident NIR pulses (Fig. 1c). A step size of 300 as was chosen to resolve short-lived features around time overlap. To examine longerlived features, the step size was increased to $1 \mathrm{fs}$ and $3 \mathrm{fs}$ at XUV-NIR delays of $130 \mathrm{fs}$ and 312 fs, respectively. The energy axis of the CCD camera was calibrated daily using atomic transition line data available from the National Institute of Standards and Technology (NIST) for the Rydberg states of argon. Except where noted, the data are presented in terms of absorbance:

$$
\mathrm{A}=-\log _{10}\left(\frac{I}{I_{0}}\right)
$$

where $I$ is the light transmitted through the krypton sample at a given XUV-NIR delay and

$I_{0}$ is a reference spectrum taken without krypton gas in the sample cell before each delaydependent scan. Absorption features have positive absorbance values while emission features from wave-mixing processes have negative absorbance values. For wave-mixing signals spatially isolated from the incident XUV pulse, $I_{0}$ comprises the dark counts from the CCD plus any residual scattered light from the XUV and NIR arms. This signal presentation mode is chosen to emphasize the weaker wave-mixing signals and indicate that they originate explicitly from newly generated light.

\section{Results}

\section{A. Spatial Isolation of Resonance-Enhanced Wave-Mixing Emission Signals}

A noncollinear geometry between a short XUV pulse train and two few-cycle NIR pulses results in background-free wave-mixing signals in krypton from the $4 s^{2} 4 \mathrm{p}^{5}\left({ }^{2} \mathrm{P}_{1 / 2}\right) n l$ states above the first IP $(14.0 \mathrm{eV})$. These states can autoionize to form the $\mathrm{Kr}^{+}{ }^{2} \mathrm{P}_{3 / 2}$ state. To generate wave-mixing signals that probe these autoionizing states, the broadband XUV pulse train (13.5 to $16.0 \mathrm{eV}$ ) induces a coherent superposition between the ground state and a manifold of bound and autoionizing states that decays with the dephasing time (polarization lifetime) of the constituent states. $^{24}$ The addition of two moderately intense, noncollinear NIR pulses produces wave-mixing signals that emit at a wavevector dependent upon the sum of the initial XUV ( $\left.k_{X U V}\right)$ and NIR $\left(k_{N I R 1}, k_{N I R 2}\right)$ wavevectors.

An X-ray CCD camera image taken at temporal overlap of all three pulses is shown in Figure 2a. The image comprises several bands, each of which results from one or more phase-matching conditions illustrated in Fig. 2b. The spatial dimension of the image is reported in divergence angle relative to the incident XUV beam. For each photon of the wave-mixing pathway resonant with an electronic transition in the atom, the intensity of the emitted signal is enhanced. All onephoton dipole-forbidden $n p$ (dark) states accessible by one NIR photon from the $n s / d$ autoionizing states are located below the first IP.

In the band centered at 0 mrad, positive absorbance values indicate the linear absorption of high harmonic XUV light by atomic resonances both above and below the first IP. At time overlap, the XUV-induced polarization is perturbed by the NIR pulses, leading to a variety of spectral effects, including spectral broadening and modification of absorption lineshapes, shifting of atomic resonances, and depletion of features. ${ }^{2,4,25,26}$ As shown by the phase-matching diagrams in Figure $2 \mathrm{~b}$, the spectrum at 0 mrad will also exhibit contributions from multiphoton wave-mixing pathways that terminate without an overall change of the emission angle such that 
$k_{X U V}-k_{N I R 1}+k_{N I R 1} \approx k_{X U V}$. The delay-dependence of these features will be discussed in Section IIIC.

A distinct band of strong negative absorbance (emission) features is observed below the harmonic axis from -2.5 to $-4.3 \mathrm{mrad}$ at the bright state energies. These features originate from wave-mixing pathways that employ photons from both NIR arms (NIR 1 and NIR 2). As shown in Fig. 2b, both third ( $k_{4 W M}=k_{X U V}-k_{N I R 1}+k_{N I R 2}$ ) and fifth (

$k_{6 W M}=k_{X U V}-k_{N I R 1}+k_{N I R 1}-k_{N I R 1}+k_{N I R 1}$ ) order processes can contribute to this band. For third order processes dependent on both NIR pulses, wave-mixing signals emit at divergence angles,

$\phi_{i}$, approximately equal to the following expression:

$$
\phi_{i} \approx \frac{v_{N I R 1} \theta_{N I R 1}+v_{N I R 2} \theta_{N I R 2}}{v_{X U V}}
$$

where $v_{N I R 1}$ and $v_{\text {NIR } 2}$ are the frequencies of the two relevant NIR interactions and $v_{X U V}$ is the frequency of the initial XUV excitation. For example, as shown in Fig. 2b, one of the wave-mixing pathways that gives rise to the signal at $14.26 \mathrm{eV}$ involves one XUV photon from the ground state to the $\left({ }^{2} \mathrm{P}_{1 / 2}\right) 9 \mathrm{~s}$ bright state $(14.26 \mathrm{eV})$ and two NIR photons resonant with the $\left({ }^{2} \mathrm{P}_{1 / 2}\right) 6 \mathrm{p}$ dark state $(1.5 \mathrm{eV})$. Using the measured NIR crossing angles of 18 and $13 \mathrm{mrad}$ and Eqn. (4), this pathway emits at a divergence angle of $-3.3 \mathrm{mrad}$, which is consistent with the experimentally observed central divergence angle of $-3.4 \mathrm{mrad}$. Discrepancies between observed and calculated emission angles can be attributed to error in the measured crossing angles and the presence of multiple different wave-mixing pathways that emit at slightly different angles. In the transient grating picture of wave-mixing, these signals are described as the first diffraction order. ${ }^{27,28}$ For simplicity, because third order signals should be more intense than fifth order signals, we refer to this band of features as the four-wave mixing signal.

A weaker band of wave-mixing signals can be observed between -6.1 and -7.8 mrad. As shown in Fig. $2 b$, these features primarily correspond to fifth order wave-mixing processes that employ one XUV photon and two photons from each NIR pulse (

$\left.k_{6 W M}=k_{X U V}-k_{\text {IIR } 1}+k_{\text {NIR1 }}-k_{\text {NIR1 }}+k_{\text {IIR } 1}\right)$. The divergence angle expected for these processes can be calculated using a relationship similar to Eqn. (4):

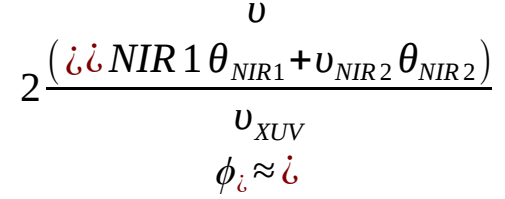

For a five photon process involving one $14.26 \mathrm{eV}$ photon and four $1.5 \mathrm{eV}$ photons, the calculated divergence angle is $-6.6 \mathrm{mrad}$. This band of fifth order features is referred to as the six-wave mixing signal.

\section{B. Delay-Dependent Wave-Mixing Emission from Autoionizing States in Krypton}

The temporal profile of the bound and autoionizing states probed can be accessed by integrating over the angular range of the four-wave mixing signals on the CCD camera image (Fig. 2a, middle band). Plotting the result as function of the delay between the XUV pulse train and the two time-coincident NIR pulses reveals information about the evolution of the XUV-induced coherence. Fig. 3a shows a false color plot of the four-wave mixing signal as a function of 
photon energy and XUV-NIR delay. Wave-mixing signals appear as negative absorbance (emission) features at the energies of atomic resonances. High-resolution photoabsorption linewidth measurements previously determined the lifetimes of the first $d$ - and $s$-character states above the IP, $\left({ }^{2} \mathrm{P}_{1 / 2}\right) 6 \mathrm{~d}$ and $\left({ }^{2} \mathrm{P}_{1 / 2}\right) 8 \mathrm{~s}$, to be $23.5 \mathrm{fs}$ and $949.5 \mathrm{fs}$ respectively, indicating that the $d$ character states are better coupled to the underlying continuum. ${ }^{29}$ As expected from examining the overlap integral, the lifetimes of autoionizing states lengthen with principal quantum number, $n$, and therefore with increasing energy. The spectral resolution is insufficient to fully resolve individual wave-mixing signals emitting from adjacent $n d / n s$ states as well as from the finely spaced states approaching the IPs.

Just below the first IP at $14.0 \mathrm{eV}$, wave-mixing features emitting from the bound states do not decay appreciably within the timeframe of the experiment. In contrast, wave-mixing signals emitting from the spectrally overlapped long-lived $n s$ and short-lived $n d$ autoionizing states above the IP exhibit visible decays. At each resonance, an intense, relatively short-lived feature can be distinguished from weaker, longer-lived quantum beat oscillations that persist for the entirety of the experiment. This short-lived feature is approximately five times more intense than the longer-lived component. Notably, the duration of the shorter-lived component increases with the principal quantum number of the emission state, following the known trend for autoionizing state lifetimes. The quantum beat oscillations that underlie both the short and long time components in the time-domain spectrum arise from the interference of wave-mixing pathways, as described in more detail below. Constituent pathways can be identified from the oscillation frequencies as determined by a Fourier transform along the delay axis (Fig. 3b). Strong, distinct features appear at $0.067 \mathrm{eV}, 0.105 \mathrm{eV}, 0.176 \mathrm{eV}$, and $0.28 \mathrm{eV}$ in the lower-lying states.

To assess the short-lived time component, three wave-mixing features at the energies of $14.1 \mathrm{eV}$, $14.26 \mathrm{eV}$, and $14.37 \mathrm{eV}$ are fit to a convolution of the instrumental response and a biexponential decay using a Levenberg-Marquardt least squares algorithm (Fig. 4). Given that the long-lived quantum beats endure well over an order of magnitude longer than the short-lived features, the long-time component was fixed at an arbitrarily long value. Varying the value of the long time constant between 500 fs and 1 ns did not significantly alter the value of the short-time constant. This fitting procedure yields decays of $22 \pm 8 \mathrm{fs}, 33 \pm 6 \mathrm{fs}$, and $49 \pm 6 \mathrm{fs}$ for the shortlived components of the features emitting from the $\left({ }^{2} \mathrm{P}_{1 / 2}\right) 6 \mathrm{~d} / 8 \mathrm{~s},\left({ }^{2} \mathrm{P}_{1 / 2}\right) 7 \mathrm{~d} / 9 \mathrm{~s}$, and $\left({ }^{2} \mathrm{P}_{1 / 2}\right) 8 \mathrm{~d} / 10 \mathrm{~s}$ states respectively, verifying the qualitative observation that the duration of the short-lived component increases with the principal quantum number of the reporter state.

Windowing the four-wave mixing spectrum to include only the first 130 fs after time overlap emphasizes the dynamics of the short-lived component (Fig. 5a). In the dense band of emission approaching the second ionization potential at $14.63 \mathrm{eV}$, the frequency of the dominant quantum beat increases with energy, creating hyperbolic band structures. As shown in Fig. 5b, the Fourier transform of just these early delays (-20 fs $-130 \mathrm{fs}$ ) is visibly distinct from the transform that encompasses longer delays (Fig. 3b). The origin of these differences is covered in Section IVA. The majority of features in Fig. 5b fall on lines with unity slope and an intercept at the energy of one of the lower lying $\left({ }^{2} \mathrm{P}_{1 / 2}\right)$ nd states. As in the full transform, these features must originate from the interference of two multiphoton pathways in the wave-mixing spectrum. 
Higher order six-wave mixing signals are captured by integrating between -6.1 and -7.8 mrad on the CCD camera image in Fig. 2a. A false color plot demonstrating the evolution of the six-wave emission as a function of photon energy and XUV-NIR delay is shown in Fig. 6a. Although the signal-to-noise ratio is lower, the isolated fifth order wave-mixing features exhibit dynamics equivalent to those observed for previously described four-wave mixing signals. However, sixwave mixing emission emerges at later XUV-NIR delays than four-wave mixing emission. As shown in Fig. 6b, the six-wave mixing signal emitting from the $\left({ }^{2} \mathrm{P}_{1 / 2}\right) 6 \mathrm{~d} / 8$ s autoionizing state is delayed by $1.8 \pm 0.4$ fs relative to the corresponding four-wave mixing signal. A similar delay was seen in $\mathrm{He}^{22}$ and is discussed further in Section IVB.

\section{Comparison to Transient Absorption of Autoionizing States in Krypton}

Integrating over the absorption band situated between 2 and -1.2 mrad on the CCD camera image yields an attosecond transient absorption measurement of the autoionizing state dynamics of $\mathrm{Kr}$ (Fig. 7a). Here, positive (absorption) features arise at the energies of the autoionizing states. Substantial energy shifts of the resonances around pulse overlap and adjacent bands at later delays indicated in ellipses i and ii can be attributed to the AC Stark effect, which gives rise to a laser-imposed phase shift that accumulates over the NIR pulse duration. ${ }^{22,25,26,30,31}$ In addition to the AC Stark effects, evidence of wave-mixing processes can be observed at early delays through quantum beat oscillations highlighted in ellipse iii and described in detail in previous publications. ${ }^{25}$ As in the wave-mixing spectra, the quantum beats approaching the second IP increase in frequency with photon energy, creating hyperbolic band structures. The most prominent features in the corresponding Fourier transform appear along lines of unity slope that intercept the $y$-axis at the energies of bright state resonances (Fig. 7b). The delay dependence extending to nearly 1 ps and the corresponding Fourier transform are provided as Supplementary Fig. 2.

\section{Discussion}

\section{A. Explanation of Experimentally Observed Wave-Mixing Signals}

The results of these noncollinear wave-mixing experiments with subfemtosecond XUV and fewcycle NIR pulses conclusively indicate that nonlinear XUV spectroscopies can access ultrafast decays of electronic excited states with notable advantages over transient absorption. From the CCD camera image alone (Fig. 2a), it is clear that the pulses employed in these experiments supply sufficient intensity to induce wave-mixing from the $4 s^{2} 4 \mathrm{p}^{5}\left({ }^{2} \mathrm{P}_{3 / 2}\right) n l$ and $\left({ }^{2} \mathrm{P}_{1 / 2}\right) n l$ states of $\mathrm{Kr}$, which exhibit a larger range of photoabsorption cross sections and decay rates than previously studied systems. Particularly notable is the observation of spatially isolated wavemixing signals at the energies of the spectrally-broad XUV-generated autoionizing states above the first IP. The time and frequency-domain spectra of these emission signals reveal a global view of autoionization dynamics occurring above the first IP in this system (Fig. 3a).

Despite substantial quantum beat oscillations indicative of contributions from multiple different states to each emission signal, the duration of the intense, short-lived component of the wavemixing features increases with the principal quantum number of the reporter (emitting) state, following the trend expected for the Rydberg state lifetimes. As shown in Table I, the 
experimentally measured decay constants for the short-lived components of the $\left({ }^{2} \mathrm{P}_{1 / 2}\right) 8 \mathrm{~s} / 6 \mathrm{~d}$, 9s/7d and 10s/8d wave-mixing features agree favorably with the population lifetimes of the $\left({ }^{2} \mathrm{P}_{1 / 2}\right) 6 \mathrm{~d}, 7 \mathrm{~d}$ and $8 \mathrm{~d}$ Rydberg states ${ }^{29}$. While more disruptive quantum beat oscillations preclude quantitative fits of higher photon energy features, the lifetimes retrieved for these lower energy emission signals suggest that the short-lived time component can be attributed to wave-mixing pathways probing the $\left({ }^{2} \mathrm{P}_{1 / 2}\right)$ nd autoionizing states. The relationship between the measured wavemixing emission lifetime and that of the $\left({ }^{2} \mathrm{P}_{1 / 2}\right)$ nd reporter state is noteworthy as it indicates that a single wave-mixing pathway dominates over other pathways that also contribute to the emission signal.

Fourier analysis of the wave-mixing emission signals can provide insight into the wave-mixing pathways that contribute to the delay dependence of the emitted signals. The Fourier transform of the time-domain data including XUV-NIR delays up to 965 fs largely provides evidence of coherences from the long-lived $n s$ autoionizing states with lifetimes on the scale of picoseconds (Fig. 3b). For example, the prominent $0.176 \mathrm{eV}$ frequency peak obtained from the $\left({ }^{2} \mathrm{P}_{1 / 2}\right) 8 \mathrm{~s}$ emission signal at $14.10 \mathrm{eV}$ (circled in Fig. 3b) can be assigned to the simultaneous XUV excitation of the $8 \mathrm{~s}(14.10 \mathrm{eV})$ and $9 \mathrm{~s}(14.27 \mathrm{eV})$ autoionizing states. The XUV-induced coherence of these two states is coupled by two NIR photons to the $\left({ }^{2} \mathrm{P}_{1 / 2}\right) 8$ s reporter state via a dark state of $p$ - or $f$-electronic character (Fig. 8a). The broad bandwidth of both the XUV and NIR pulses allows not only for the XUV-induced coherent excitation of a manifold of excited states, but also for extensive dark-state mediated coupling of states within in the $\sim 0.5 \mathrm{eV} \mathrm{NIR}$ bandwidth. Other prominent Fourier peaks at low photon energies can be assigned to the XUVinduced coherence of the $10 \mathrm{~s}$ and $11 \mathrm{~s}$ states $(0.067 \mathrm{eV})$, 9s and $10 \mathrm{~s}$ states $(0.105 \mathrm{eV})$, and 8 s and 10 s states $(0.28 \mathrm{eV})$. These features demonstrate the extensive coupling between Rydberg states that occurs within this system, emphasizing that the observed wave-mixing signals probe the evolution of multiple XUV-excited states.

Fourier analysis of only early delays ( $130 \mathrm{fs}$ ) reveals contributions from the shorter-lived nd autoionizing states (Fig. 5b). The hyperbolic band structures in Fig. 5a generate peaks in the photon-energy resolved Fourier transform that fall on lines with unity slope, which are highlighted by white lines in Fig. 5b. All features along the white lines arise from wave-mixing pathways that couple the state at the intercept with the final state at which the feature is observed. Here, the lines intercept the y-axis at the energies of the nd states preferentially. To produce such lines, the second interfering pathway is best described by a wave-mixing process with two degenerate NIR photons that couples the reporter state with itself. An example of two interfering pathways that lead to a peak in the early-delay Fourier transform are provided as Pathways \#1 and \#2 in Fig. 8b. Pathways with two degenerate NIR photons like Pathway \#2 appear to dominate the total emitted signal from the manifold of autoionizing states at early times, leading to the hyperbolic band structures and the intense, short-lived component observed in the time-domain wave-mixing signals, as will be discussed further in Section VB.

Moreover, because of large contributions from these NIR-degenerate pathways, the delaydependent wave-mixing spectra provide more insight into the underlying excited state dynamics than a traditional transient absorption measurement. In previous attosecond transient absorption studies, AC-Stark effects were shown to limit the accuracy of lifetime measurements to only lineouts taken exactly on-resonance. ${ }^{4}$ As shown in Fig. 7a, the severity of the AC Stark shift of 
the resonances around pulse overlap precludes any lineout measurement of the excited state lifetime, particularly of the short-lived $\left({ }^{2} \mathrm{P}_{1 / 2}\right)$ nd states. Without resolvable decays, the disappearance of quantum beat oscillations provides the most convincing evidence of autoionization in the transient absorption spectrum. The quantum beats produce peaks that appear along unity slope lines in the Fourier transform reminiscent of the features observed in the transform of the noncollinear wave-mixing signals at early delays (Fig. 7b). These lines are interpreted as the interference of a one-photon, linear response pathway (Fig. 8c, Pathway \#1) and a three photon, wave-mixing pathway (Fig 8c, Pathway \#2) that emit at the state in which the beat occurs. The interference of one and three photon pathways is discussed extensively in the transient absorption literature. ${ }^{25}$

\section{B. General Characteristics of Wave Mixing with Ultrashort Pulses}

The conspicuous differences between transient absorption and wave-mixing spectra can be attributed to the presence or absence of the XUV-excitation field. In transient absorption experiments, NIR-induced features, including those generated by the AC Stark effect and wavemixing pathways, interfere with the incident high harmonic XUV light. The XUV field thus acts as a reference field, allowing for phase-sensitive heterodyne detection ${ }^{9}$. Using a square law detector, the intensity of the detected signal can be described by the following relationship:

$$
I_{\text {signal }}=\left|E_{X U V}+E_{E m i t}\right|^{2}=\left|E_{X U V}\right|^{2}+\left|E_{E m i t}\right|^{2}+2 R\left(E_{E m i t} E_{X U V}^{i}\right)
$$

where $I_{\text {signal }}$ is the intensity of the detected signal, $E_{X U V}$ is the electric field of the incident XUV pulse train, and $E_{\text {Emit }}$ is the electric field of generated polarization signal. When the incident XUV field is present, $E_{\text {Emit }}$ includes both the first order polarization and any higherorder polarization fields. In a perturbative picture, the intensity of the incident XUV field can be assumed to be greater than the generated light. The intensity detected by the CCD camera can then be described by the following expression:

$$
I_{\text {signal }}=\left|E_{X U V}+E_{\text {Emit }}\right|^{2} \approx\left|E_{X U V}\right|^{2}+2 R\left(E_{\text {Emit }} E_{X U V}^{i}\right)
$$

The time-domain dynamics of the detected field in this self-heterodyned detection scheme are dominated by the cross-term. ${ }^{9}$

For wave-mixing measurements in a noncollinear geometry, the reference XUV field does not overlap spatially with generated wave-mixing signals, resulting in a homodyne detection scheme. In optical homodyne detection, the square modulus of the incident field and the cross term can be ignored. The detected intensity can therefore be adequately described by only the square modulus of the emitted field, which only includes wave-mixing fields and is proportional the polarization response function, $R^{(3)}\left(t_{3}, t_{2}, t_{1}\right)$ :

$$
\left|E_{E m i t}\right|^{2}=\left|E_{W M}\right|^{2} \propto\left|R^{(3)}\left(t_{3}, t_{2}, t_{1}\right)\right|^{2}
$$

where $E_{W M}$ is the electric field of the wave-mixing signals. The time convention for $t_{1}$,

$t_{2}$, and $t_{3}$ is defined in Fig. 9a. The relevant components of the wave mixing response function for a single, arbitrary four-wave mixing pathway (Fig. 9b) utilized in our experiment can be defined as:

$$
R^{(3)}\left(t_{3}, t_{2}, t_{1}\right) \propto \mu_{01}^{2} \mu_{12}^{2} e^{-i\left(\omega_{1}-\omega_{0}\right) t_{1}-\frac{t_{1}}{T_{2}}}
$$


where $\mu_{01}$ and $\quad \mu_{12}$ are the transition dipoles associated with the transition between states $i 0$ and $i 1$ and between states $i 1\rangle$ and $i 2$. The frequencies of states $i 0$ and i1) are described by $\omega_{0}$ and $\omega_{1}$, respectively. The decay term $T_{2}$ describes the dephasing time, which equals twice the population lifetime ( $T_{1}$ ) for a system in which dephasing due to the environment is negligible ${ }^{9}$. A more detailed derivation of the third-order response function using diagrammatic perturbation theory is provided as Supplementary Note 3. In heterodyne detection schemes dominated by the cross-term, the observed decay is equal to $T_{2}$. To demonstrate the impact of this homodyne detection scheme on a time-domain signal, the response function is incorporated into the Eqn. (8) as follows:

$$
\left|E_{W M}\right|^{2} \propto \mid \mu_{01}^{2} \mu_{12}^{2} e^{\left.-i \mid \omega_{1}-\omega_{0}\right) t_{1}-\left.\frac{t_{1}}{T_{2}}\right|^{2}} \propto \mu_{01}^{4} \mu_{12}^{4} e^{-2 \frac{t_{1}}{T_{2}}}
$$

The time-integrated response of the detector over $t_{3}$ is not detailed here. Note that, without contributions from the cross-term, frequency-dependent terms that result in AC Stark hyperbolic side bands are eliminated by the square modulus. Furthermore, in this homodyne geometry, the measured decay equals the excited state lifetime as $T_{1}=\frac{T_{2}}{2}$.

The above treatment applies for the emission of a single wave-mixing pathway. However, because of the broad bandwidths of the pulses employed in these experiments, the time evolution of individual emission signals reflects the contributions of multiple different wave-mixing pathways. ${ }^{21}$ Pathways like those in Fig. 8a-b interfere, resulting in quantum beat oscillations, and should collectively dictate the decay of the emission feature. As demonstrated by the perturbative simulation shown in Supplementary Note 3, significant contributions from multiple pathways with nondegenerate NIR photons, like Pathway \#1 in Fig. 8b, effectively eliminate lifetime variations between wave-mixing signals emitting from different reporter states. However, the experimental wave-mixing spectrum does exhibit reporter-state dependent lifetime variations. The duration of the short-lived component visibly increases with the principal quantum number of the reporter state. Moreover, the measured lifetimes track those of the Rydberg states coupled to the reporter states by a NIR-degenerate pathway like Pathway \#2 in Fig. 8b. Supported by the early-delay Fourier transform (Fig. 5b), these NIR-degenerate pathways appear to be systematically enhanced relative to other pathways for the entire manifold of autoionizing states, allowing for the preferential coupling of the reporter states to themselves. The enhancement of NIR-degenerate pathways therefore leads to reporter-state dependent variations in the delaydependence of these wave-mixing signals.

While not yet fully characterized in this system, the enhancement of NIR-degenerate pathways may potentially be explained by effects that cannot be fully captured by a perturbative picture of wave mixing. In a perturbative picture for a fully-resonant wave-mixing process, the interaction of each pulse with the medium is treated as the linear absorption or emission of a photon from an eigenstate of the atom. For the system studied here, the emission of a broadband ( $0.5 \mathrm{eV})$ NIR photon projects the XUV-induced coherence onto dark states located below the IP. The simultaneous interaction of a second NIR photon generates resonance-enhanced wave-mixing signals that emit from all states within the bandwidth of the NIR pulse, including the autoionizing states above the first IP. However, features suggesting significant deviations from a perturbative wave-mixing regime can be identified in this system. Previous experiments on long- 
lived Rydberg and dressed states in atomic helium have demonstrated that phase gratings due to the nonresonant AC Stark effect are instrumental in the formation of wave-mixing signals in this experimental geometry, leading to delays in emergence of higher diffraction orders. ${ }^{22}$ As shown in Fig. 6b, the six-wave mixing signal emitting at the energy of the $\left({ }^{2} \mathrm{P}_{1 / 2}\right) 6 \mathrm{~d} / 8 \mathrm{~s}$ autoionizing state is delayed by $1.8 \pm 0.4$ fs relative to the four-wave mixing signal, indicating that a purely perturbative wave mixing picture cannot adequately capture all the features observed of this experiment.

In previous literature, the AC-Stark effect has been implicated in nonperturbative wave-mixing regimes as well as in pathway enhancement ${ }^{32-35}$ and therefore may provide an attractive mechanism by which to explain the enhancement of NIR-degenerate pathways in this experiment. However, the development of theoretical models that provide a general understanding of the impact of nonperturbative effects on wave-mixing signals is currently an active area of research. ${ }^{34,36-39}$ As few nonperturbative treatments of multilevel systems are currently available, more work is necessary to clarify the role of the AC-Stark effect on the evolution of wave-mixing signals in a complex system like the one studied here. Regardless of the mechanism, this experiment suggests that the enhancement of desirable wave-mixing pathways can assist in the measurement of ultrafast lifetimes by, in essence, reducing the complexity of the wave-mixing experiments. Once fully characterized, the mechanism that allows for pathway enhancement, such as the AC-Stark effect, may ultimately be utilized as a tool to enhance or eliminate particular nonlinear processes, providing experimentalists with substantive control over signal evolution.

\section{Conclusions}

Utilizing a noncollinear geometry with a short train of subfemtosecond XUV pulses and two few-cycle NIR pulses, we directly probe the time-domain evolution of spatially isolated wavemixing signals from autoionizing states that decay on a variety of different timescales. This work not only represents the first successful generation of isolated XUV wave-mixing signals from states that decay on these timescales, but also demonstrates that wave-mixing experiments performed in a noncollinear geometry may confer significant advantages over transient absorption experiments when measuring ultrafast decays. Due to the suppression of AC Stark energy level shifting and sidebands in homodyne wave-mixing experiments without the high harmonic XUV field, lifetimes of the short-lived $\left({ }^{2} \mathrm{P}_{1 / 2}\right)$ nd autoionizing states can be extracted directly from the time-domain signal despite persistent quantum beat oscillations. The observed lifetime trend can be attributed to wave-mixing pathways coupling the reporter states to themselves that dominate at the early delays. While the origins of this pathway enhancement are not yet fully established, delays between the emergence of four and six-wave mixing emission signals due to the accumulation of an AC-Stark grating provide evidence that nonperturbative effects impact signal generation and may influence signal evolution in this system. This work suggests that the selection of desired pathways, either through pathway enhancement as shown here or via multidimensional spectroscopic techniques, can support the measurement of ultrafast lifetimes, revealing dynamics that would have otherwise been obscured by the multiplexed nonlinear response of the system.

\section{Acknowledgements}


This work was supported by the Director, Office of Science, Office of Basic Energy Sciences through the Atomic, Molecular, and Optical Sciences Program of the Division of Chemical Sciences, Geosciences, and Biosciences of the US Department of Energy at LBNL under contract no. DE-AC02-05CH11231. A.P.F. acknowledges funding from the National Science Foundation Graduate Research Fellowship Program.

\section{Supplementary Material}

See supplementary material for CMOS camera images for pulse overlap determination, a transient absorption spectrum of the $\left({ }^{2} \mathrm{P}_{1 / 2}\right) n s / d$ states over nearly a picosecond of delays, and a fully resonant, perturbative simulation of the wave-mixing spectrum.

\section{References}

${ }^{1}$ F. Krausz and M. Ivanov, Rev. Mod. Phys. 81, 163 (2009).

${ }^{2}$ A.R. Beck, D.M. Neumark, and S.R. Leone, Chem. Phys. Lett. 624, 119 (2015).

${ }^{3}$ K. Ramasesha, S.R. Leone, and D.M. Neumark, Annu. Rev. Phys. Chem. 67, 41 (2016).

${ }^{4}$ B. Bernhardt, A.R. Beck, X. Li, E.R. Warrick, M.J. Bell, D.J. Haxton, C.W. McCurdy, D.M. Neumark, and S.R. Leone, Phys. Rev. A - At. Mol. Opt. Phys. 89, 1 (2014).

${ }^{5}$ X. Li, B. Bernhardt, A.R. Beck, E.R. Warrick, A.N. Pfeiffer, M. Justine Bell, D.J. Haxton, C. William McCurdy, D.M. Neumark, and S.R. Leone, J. Phys. B At. Mol. Opt. Phys. 48, (2015).

${ }^{6}$ M. Drescher, M. Hentschel, R. Kienberger, M. Uiberacker, V. Yakovlev, a Scrinzi, T. Westerwalbesloh, U. Kleineberg, U. Heinzmann, and F. Krausz, Nature 419, 803 (2002).

${ }^{7}$ F. Calegari, A. Trabattoni, A. Palacios, D. Ayuso, M.C. Castrovilli, J.B. Greenwood, P. Decleva, F. Martín, and M. Nisoli, J. Phys. B At. Mol. Opt. Phys. 49, 142001 (2016).

${ }^{8}$ S. Mukamel, Principles of Nonlinear Optical Spectroscopy (Oxford University Press, New York, 1995).

${ }^{9}$ P. Hamm and M.T. Zanni, Concepts and Methods of 2D Infrared Spectroscopy (Cambridge University Press, Cambridge, 2011).

${ }^{10}$ T.R. Calhoun, N.S. Ginsberg, G.S. Schlau-Cohen, Y.C. Cheng, M. Ballottari, R. Bassi, and G.R. Fleming, J. Phys. Chem. B 113, 16291 (2009).

${ }^{11}$ G.S. Schlau-Cohen, T.R. Calhoun, N.S. Ginsberg, E.L. Read, M. Ballottari, R. Bassi, R. van Grondelle, and G.R. Fleming, J. Phys. Chem. B 113, 15352 (2009).

${ }^{12}$ D. Zigmantas, E.L. Read, T. Mancal, T. Brixner, A.T. Gardiner, R.J. Cogdell, and G.R. Fleming, Proc. Natl. Acad. Sci. 103, 12672 (2006).

${ }^{13}$ Y.R. Chen, Nature 337, 519 (1989).

${ }^{14}$ P.E. Ohno, S.A. Saslow, H.F. Wang, F.M. Geiger, and K.B. Eisenthal, Nat. Commun. 7, $13587 \mid$ (2016).

${ }^{15}$ C.L. Evans and X.S. Xie, Annu. Rev. Anal. Chem. 1, 883 (2008).

${ }^{16}$ A. Zumbusch, G.R. Holtom, and X.S. Xie, Phys. Rev. Lett. 82, 4142 (1999).

${ }^{17}$ W. Cao, E.R. Warrick, A. Fidler, S.R. Leone, and D.M. Neumark, Phys. Rev. A 94, 021802 (2016).

${ }^{18}$ W. Cao, E.R. Warrick, A. Fidler, D.M. Neumark, and S.R. Leone, Phys. Rev. A - At. Mol. Opt. Phys. 94, 053846 (2016).

${ }^{19}$ W. Cao, E.R. Warrick, A. Fidler, S.R. Leone, and D.M. Neumark, Phys. Rev. A 97, 023401 (2018).

${ }^{20}$ E.R. Warrick, A. Fidler, W. Cao, E. Bloch, D.M. Neumark, and S.R. Leone, Faraday Discuss. 
212, 157 (2018).

${ }^{21}$ H.J.B. Marroux, A.P. Fidler, D.M. Neumark, and S.R. Leone, Sci. Adv. 4, (2018).

${ }^{22}$ A.P. Fidler, S.J. Camp, E.R. Warrick, E. Bloch, H.J.B. Marroux, D.M. Neumark, K.J. Schafer, M.B. Gaarde, and S.R. Leone, Nat. Commun. 10, 1384 (2019).

${ }^{23}$ H. Timmers, Y. Kobayashi, K.F. Chang, M. Reduzzi, D.M. Neumark, and S.R. Leone, Opt. Lett. 42, 811 (2017).

${ }^{24}$ S. Bengtsson, E.W. Larsen, D. Kroon, S. Camp, M. Miranda, C.L. Arnold, A. L’Huillier, K.J. Schafer, M.B. Gaarde, L. Rippe, and J. Mauritsson, Nat. Photonics 11, 252 (2017).

${ }^{25}$ W. Cao, E.R. Warrick, D.M. Neumark, and S.R. Leone, New J. Phys. 18, 013041 (2016).

${ }^{26}$ M. Wu, S. Chen, S. Camp, K.J. Schafer, and M.B. Gaarde, J. Phys. B At. Mol. Opt. Phys. 49, 062003 (2016).

${ }^{27}$ A. Blouin, M.-M. Denariez Roberge, and P. Galarneau, J. Opt. Soc. Am. B Opt. Phys. 8, 578 (1991).

${ }^{28}$ E.J. Brown, Q. Zhang, and M. Dantus, J. Chem. Phys. 110, 5772 (1999).

${ }^{29}$ K. Maeda, K. Ueda, and K. Ito, J. Phys. B At. Mol. Opt. Phys. 26, 1541 (1993).

${ }^{30}$ C. Ott, A. Kaldun, P. Raith, K. Meyer, M. Laux, J. Evers, C.H. Keitel, C.H. Greene, and T. Pfeifer, Science (80-. ). 340, 716 (2013).

${ }^{31}$ S. Chen, M. Wu, M.B. Gaarde, and K.J. Schafer, Phys. Rev. A - At. Mol. Opt. Phys. 88, 033409 (2013).

${ }^{32}$ A.M. Levine, N. Chencinski, W.M. Schreiber, A.N. Weiszmann, and Y. Prior, Phys. Rev. A 35, 2550 (1987).

${ }^{33}$ W. Chalupczak, W. Gawlik, and J. Zachorowski, Phys. Rev. A 49, 4895 (1994).

${ }^{34}$ D.A. Coppeta, P.L. Kelley, P.J. Harshman, and T.K. Gustafson, 53, 925 (1996).

${ }^{35}$ C.-Y. Tai, R.T. Deck, and C. Kim, Phys. Rev. A 37, 163 (1988).

${ }^{36}$ L. Chen, E. Palacino-González, M.F. Gelin, and W. Domcke, J. Chem. Phys. 147, (2017).

${ }^{37}$ M.F. Gelin, D. Egorova, and W. Domcke, J. Phys. Chem. B 115, 5648 (2011).

${ }^{38}$ V. V. Strelkov, Phys. Rev. A 93, 1 (2016).

${ }^{39}$ J.M. Shacklette and S.T. Cundiff, J. Opt. Soc. Am. B 20, 764 (2003). 
Tables

\begin{tabular}{|c|c|c|}
\hline Reporter State & Four-Wave Mixing Lifetime (fs) & Literature Lifetime (fs) $^{\mathbf{2 9}}$ \\
\hline$\left({ }^{2} \mathrm{P}_{1 / 2}\right) 6 \mathrm{~d}$ & $22 \pm 8$ & 23.5 \\
\hline$\left({ }^{2} \mathrm{P}_{1 / 2}\right) 7 \mathrm{~d}$ & $33 \pm 6$ & 34.0 \\
\hline$\left({ }^{2} \mathrm{P}_{1 / 2}\right) 8 \mathrm{~d}$ & $49 \pm 6$ & 47.1 \\
\hline
\end{tabular}

TABLE 1. Comparison of measured four-wave mixing lifetimes with literature values. Lifetimes measured for three four-wave mixing signals in the time-domain spectrum compare favorably with lifetimes of the $\left({ }^{2} \mathrm{P}_{1 / 2}\right)$ nd reporter states determined from linewidth measurements. 


\section{Figures}
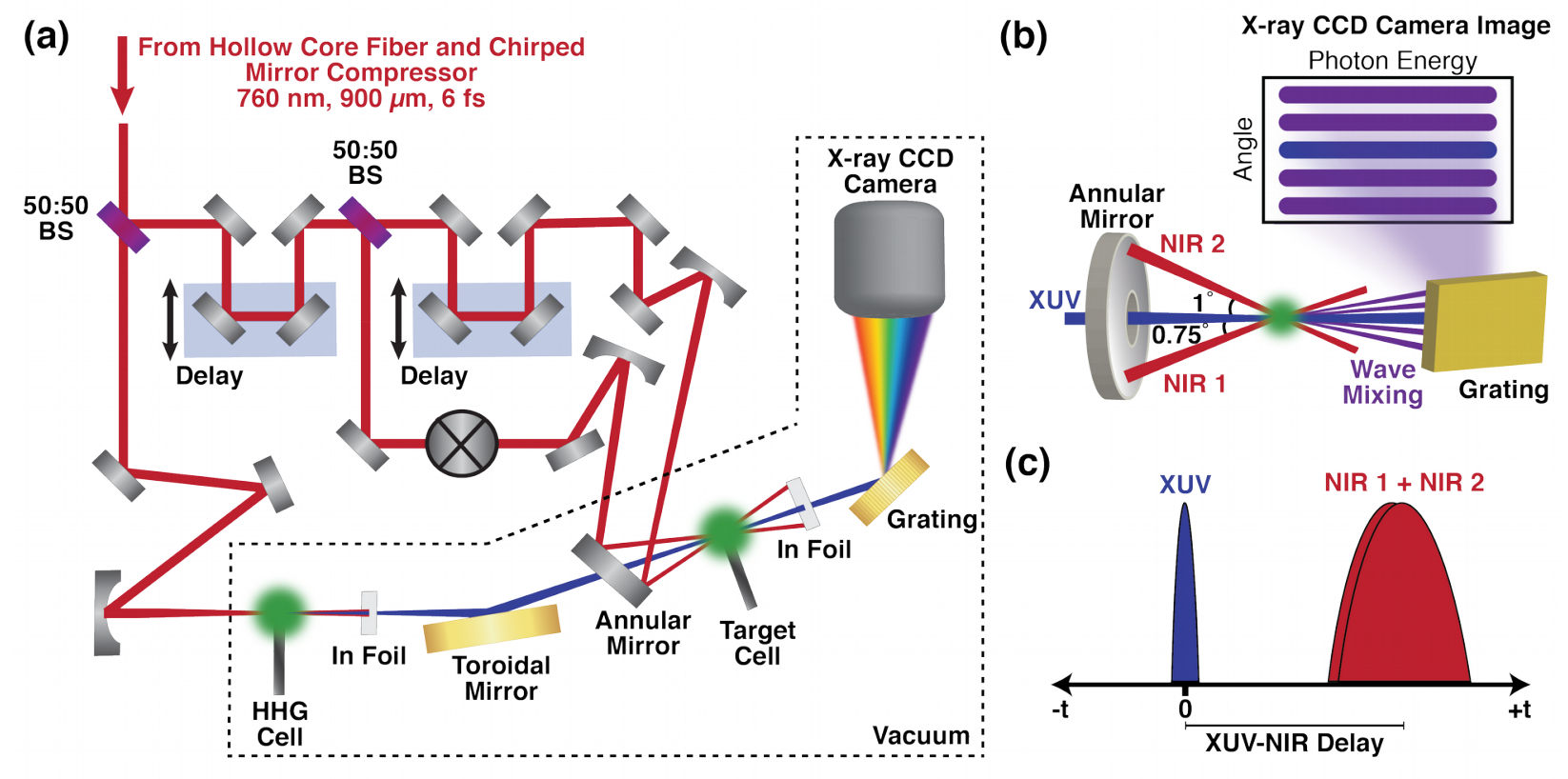

FIG 1. Experimental apparatus and pulse sequence. (a) Experimental apparatus after NIR pulse compression used for noncollinear wave-mixing experiments. (b) The interaction of the noncollinear NIR pulses with the XUV-excited sample leads to spatial separation of wavemixing signals. The NIR beam oriented below the hole mirror is labelled NIR 1 while the beam oriented above the mirror is labelled NIR 2. (c) Dynamics encoded in wave-mixing signals are measured as a function of the time delay between the XUV and time-coincident NIR pulses. Positive delays indicate that the XUV pulse precedes the NIR pulses. 

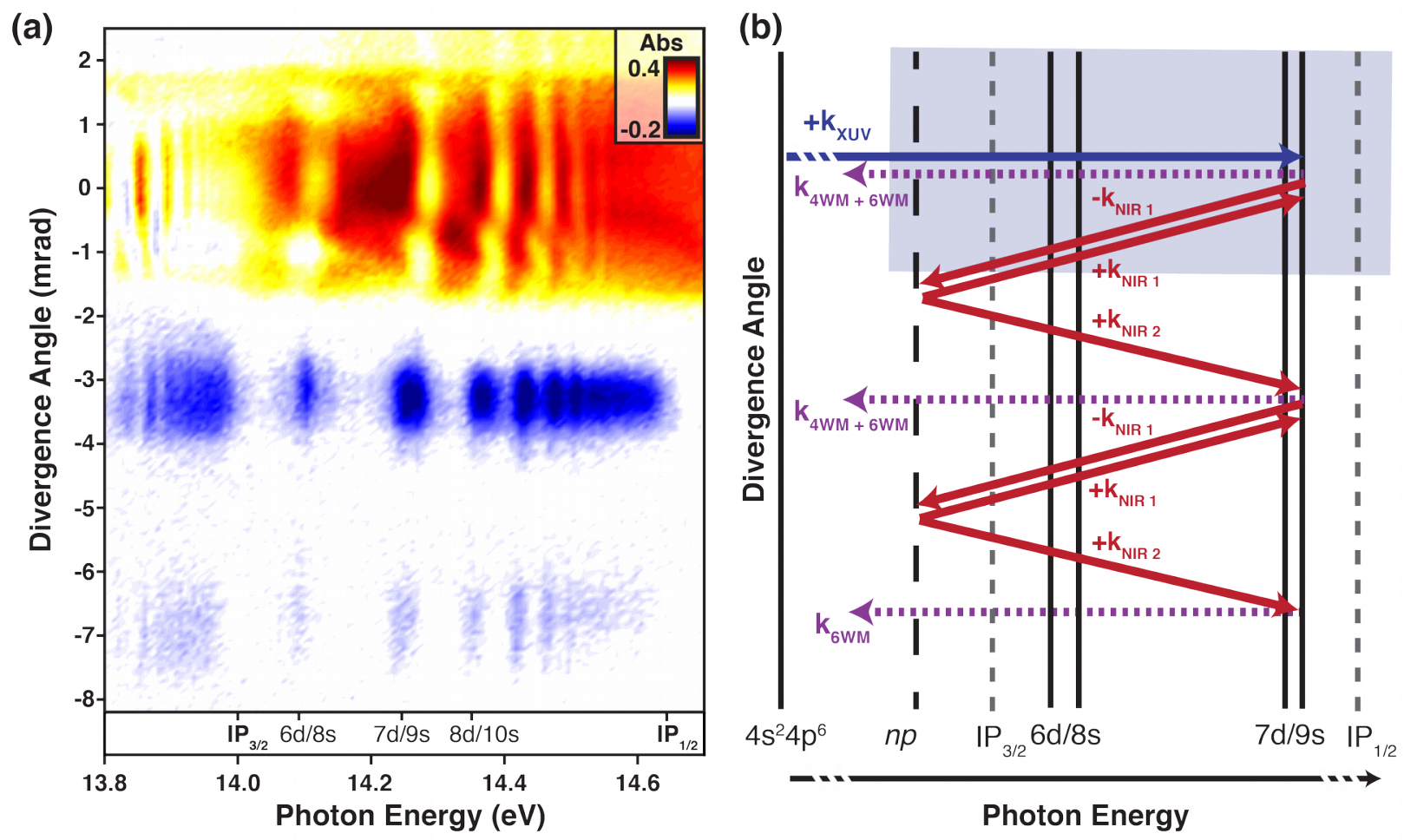

FIG 2: Spatially-dependent emission of multiple orders of wave-mixing signals. (a) An $x$-ray charge coupled device (CCD) image plotted as function of photon energy and phase-matching divergence angle exhibits three distinct bands of absorption or wave-mixing signals. The energies of the first two ionization potentials and several key autoionizing states are provided at the bottom of the image. (b) Example phase-matching diagrams show wave-mixing pathways that emit at each of the three bands at the $\left({ }^{2} \mathrm{P}_{1 / 2}\right) 7 \mathrm{~d} / 9 \mathrm{~s}$ resonance at $14.26 \mathrm{eV}$. The two bands closest to $0 \mathrm{mrad}$ contain contributions from multiple orders of wave-mixing signals. The blue, red, and dotted purple arrows indicate photons of XUV, NIR, or generated XUV wave-mixing light, respectively. 
(a)

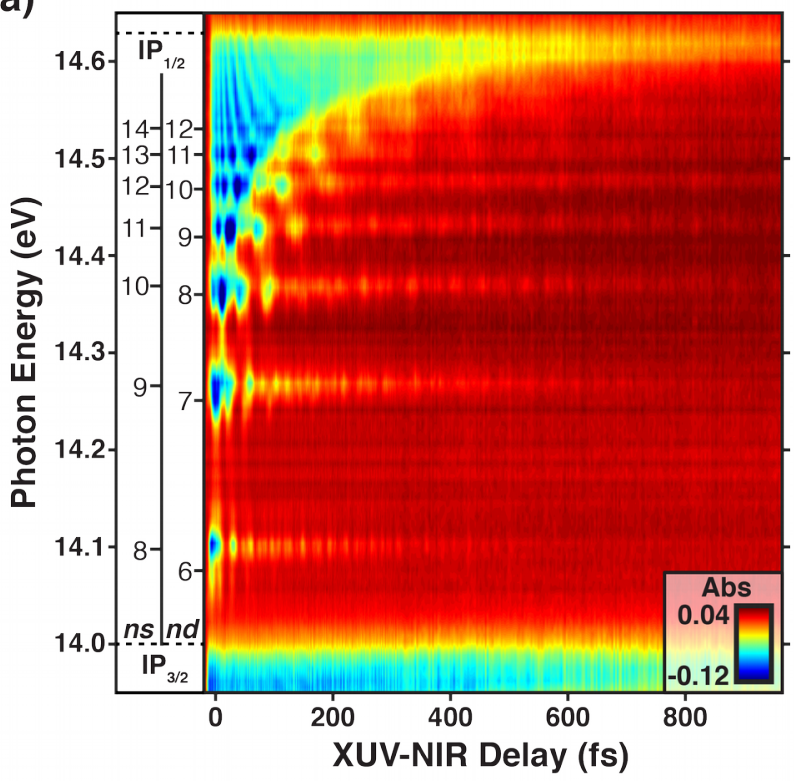

(b)

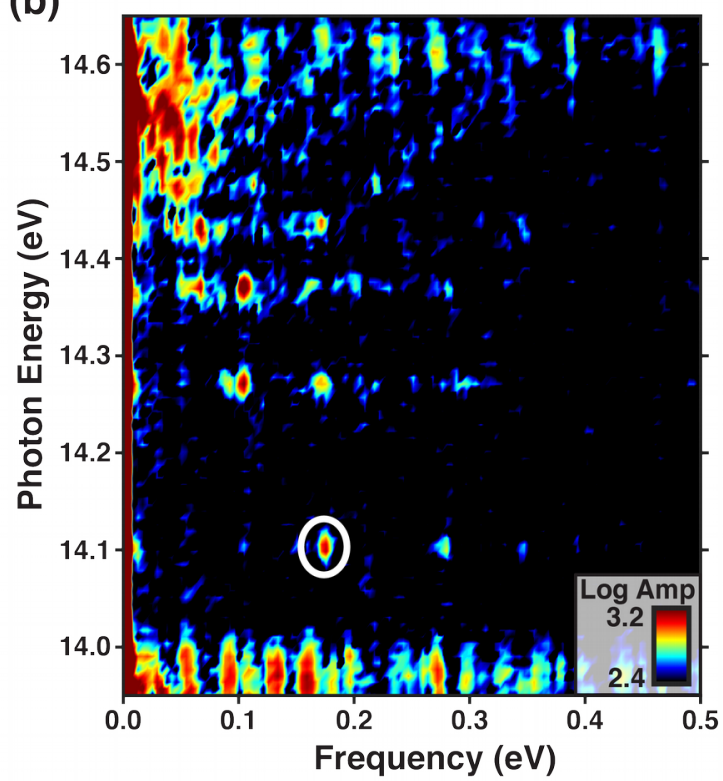

FIG 3: Four-wave mixing measurements of $\left({ }^{2} \mathbf{P}_{1 / 2}\right) n l$ autoionizing states of $\mathrm{Kr}$. (a) Emission spectrum of the four-wave mixing signals as a function of energy and XUV-NIR delay. State assignments are provided to the left of the panel. (b) Fourier analysis of the delay-dependent spectrum. The white circle highlights a feature arising from the interference of two wave-mixing pathways that will be discussed explicitly in Fig. 8. 

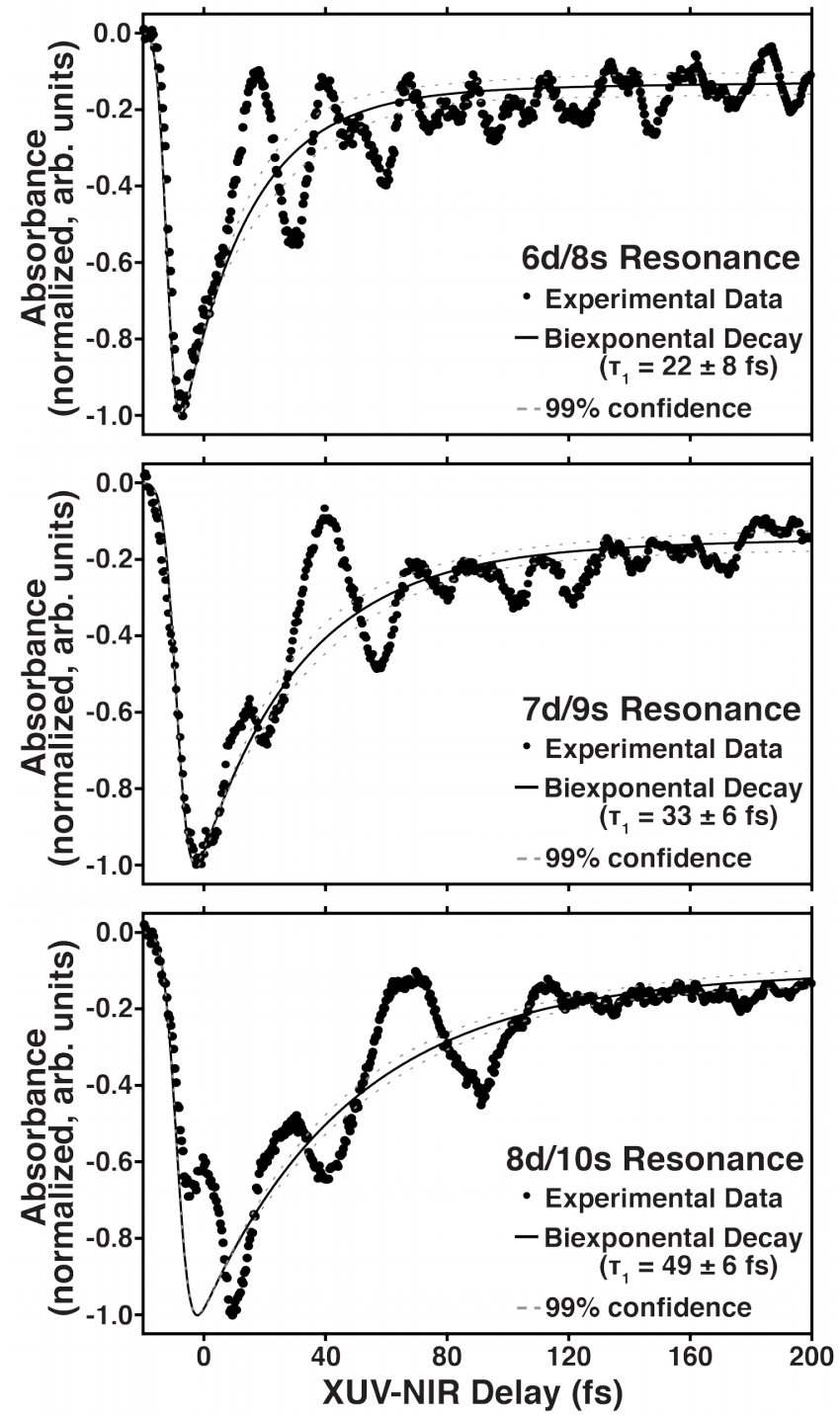

FIG 4: Lifetime analysis of ultrafast wave-mixing decays. Lineouts taken from the delaydependent four-wave mixing signal at the (a) $\left({ }^{2} \mathrm{P}_{1 / 2}\right) 6 \mathrm{~d} / 8 \mathrm{~s}$ resonance $(14.1 \mathrm{eV})$, (b) $\left({ }^{2} \mathrm{P}_{1 / 2}\right) 7 \mathrm{~d} / 9 \mathrm{~s}$ resonance $(14.26 \mathrm{eV})$, and (c) $\left({ }^{2} \mathrm{P}_{1 / 2}\right) 8 \mathrm{~d} / 10$ s resonance $(14.37 \mathrm{eV})$ are fit to biexponential decays with an arbitrary long time constant. The fit short time constant compares favorably with the excited state population lifetimes known for the $\left({ }^{2} \mathrm{P}_{1 / 2}\right) 6 \mathrm{~d},\left({ }^{2} \mathrm{P}_{1 / 2}\right) 7 \mathrm{~d}$, and $\left({ }^{2} \mathrm{P}_{1 / 2}\right) 8 \mathrm{~d}$ states, respectively (See text for details). 
(a)

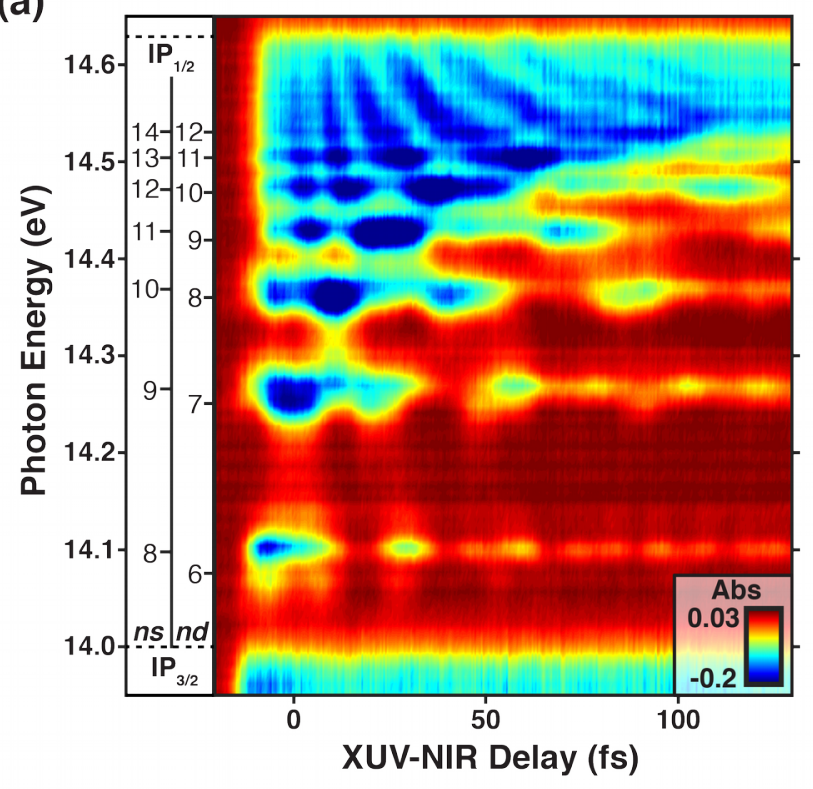

(b)

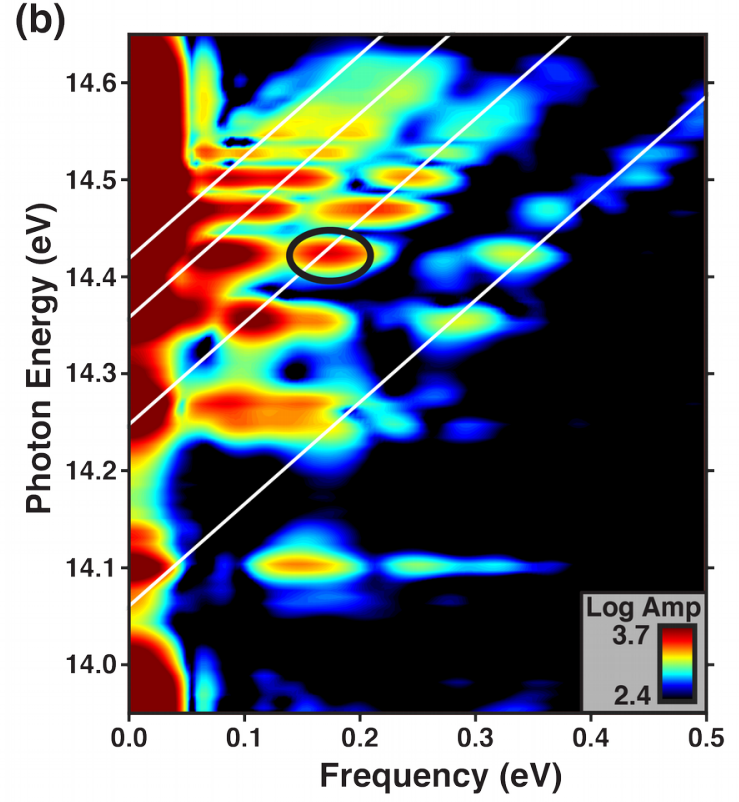

FIG 5: Early delay-dependence of four-wave mixing signals in Kr. (a) Emission spectrum of the four-wave mixing signals windowed to encompass only XUV-NIR delays from -20 to 130 fs. State assignments are provided to the left of the panel. (b) Fourier analysis of the windowed delay-dependent spectrum. The white lines have unity slope and indicate that the features along them arise in part from a wave-mixing pathway that begins with XUV excitation to the state at the intercept. The feature highlighted by the black circle will be identified in Fig. 8b. 
(a)

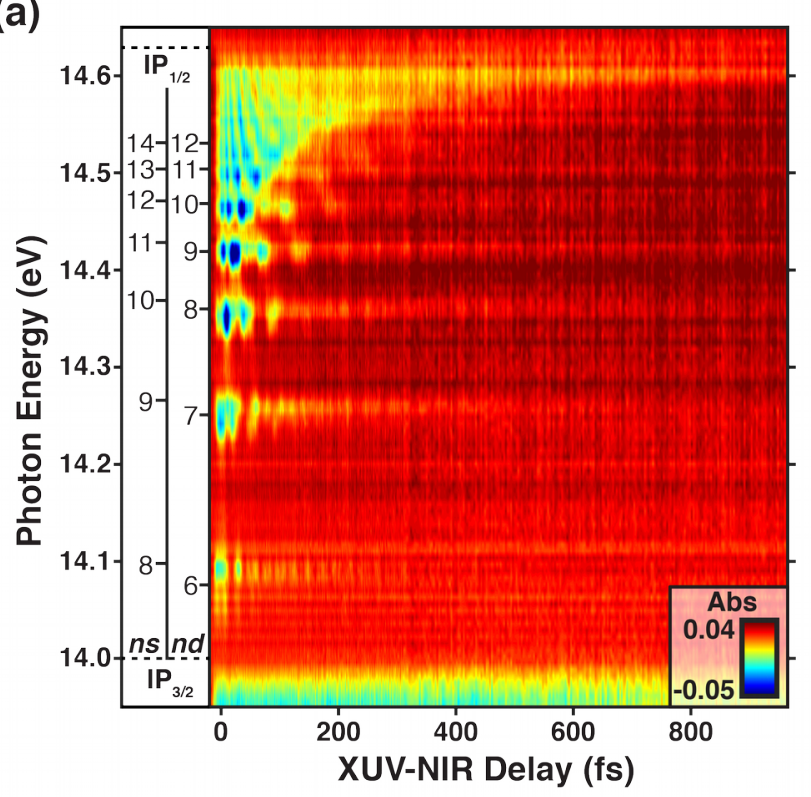

(b)

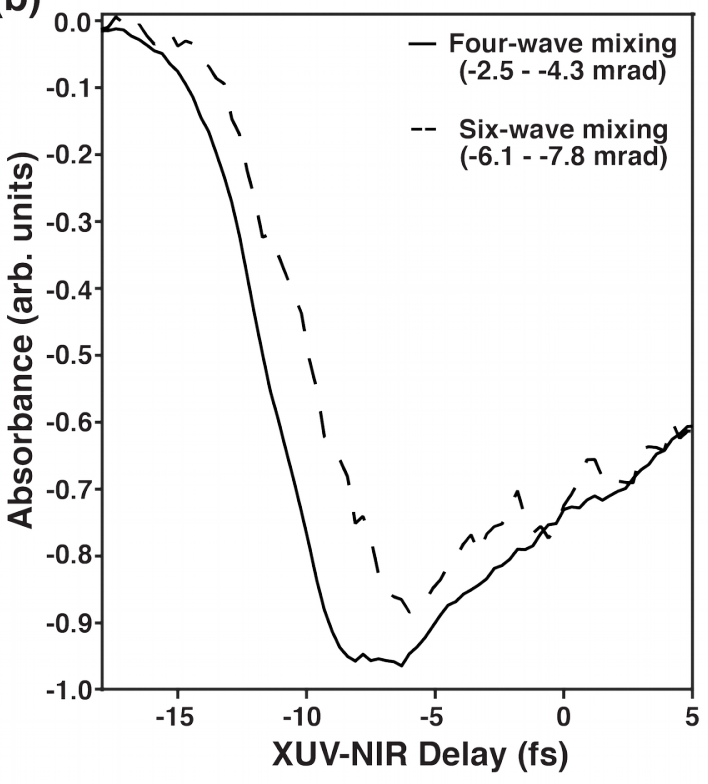

FIG 6: Six-wave mixing measurements of autoionizing states of Kr. (a) Absorption spectrum of the six-wave mixing signals as a function of energy and XUV-NIR delay. State assignments are provided to the left of the panel. (b) Lineouts of wave-mixing signals generated at the $\left({ }^{2} \mathrm{P}_{1 / 2}\right) 7 \mathrm{~d} / 9 \mathrm{~s}$ resonance $(14.1 \mathrm{eV})$ obtained by integrating over the predominately four-wave mixing emission (-2.5 - -4.5 mrad) or the six-wave mixing emission (-6.1 - -7.8 mrad) bands in the CCD camera image shown in Fig 2a. 
(a)

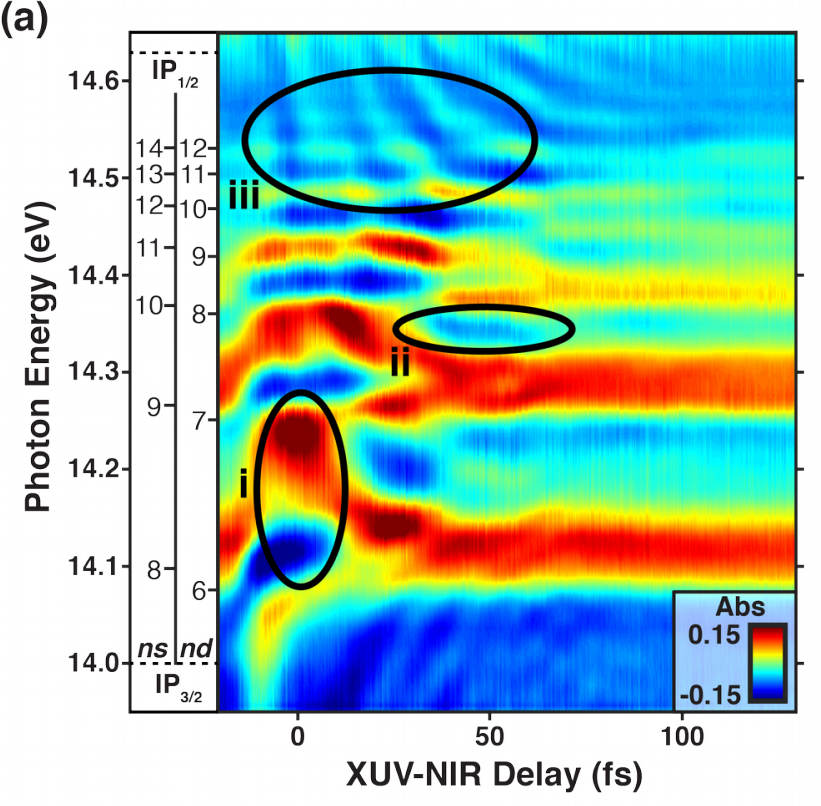

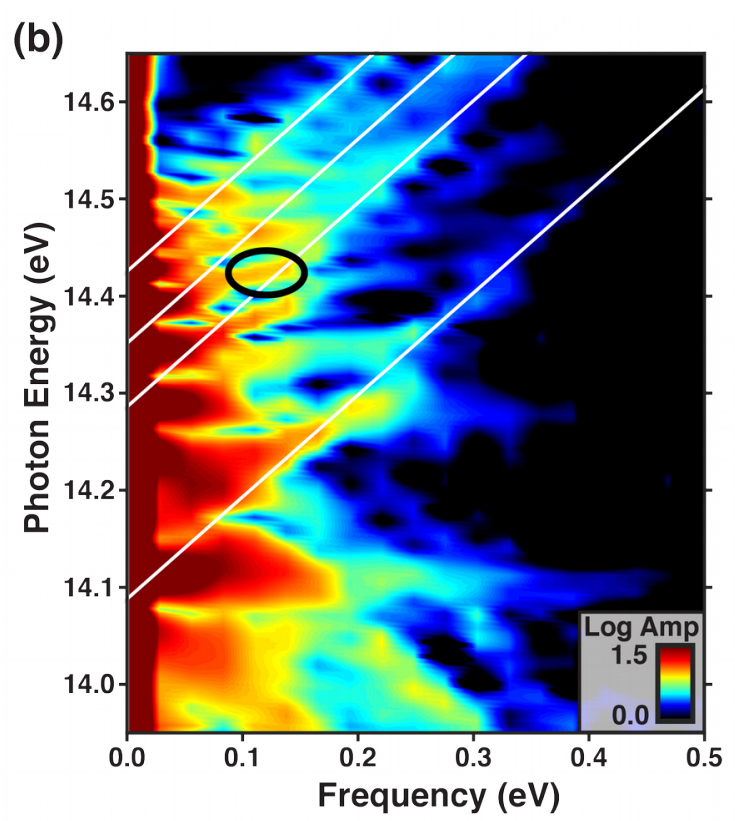

FIG 7: Transient absorption measurements of autoionizing states of $\mathbf{K r}$. (a) Absorption is plotted as a function of XUV-NIR delay and energy. States assignments are located to the left of the panel. Black ellipses indicate features of interest discussed in the text. (b) Fourier analysis of the delay-dependent spectrum. The white lines possess a unity slope and indicate that the features along them arise from the interference of the linear response and a wave-mixing pathway that begins with an XUV excitation to the state at the intercept. The feature circled in black will be discussed further in Fig. 8a. 
(a) Four-Wave Mixing Late Delays

Pathway \#1

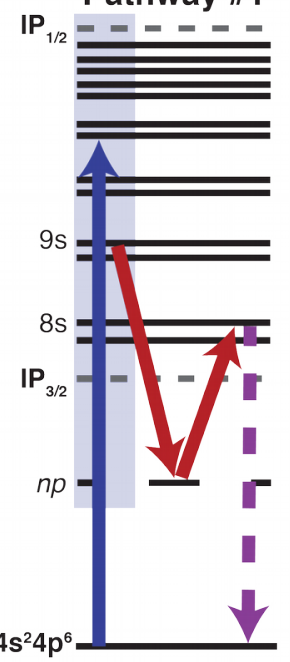

(b) Four-Wave Mixing Early Delays

Pathway \#2
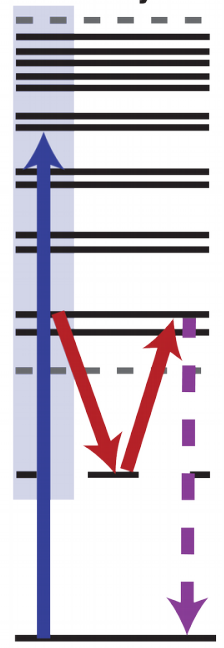

Pathway \#1

$$
\mathbf{I P}_{1 / 2}
$$

$----$.

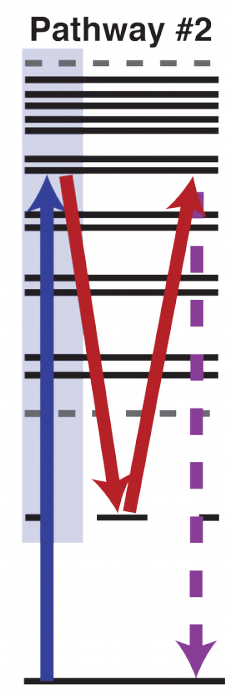

(c) Transient Absorption Early Delays

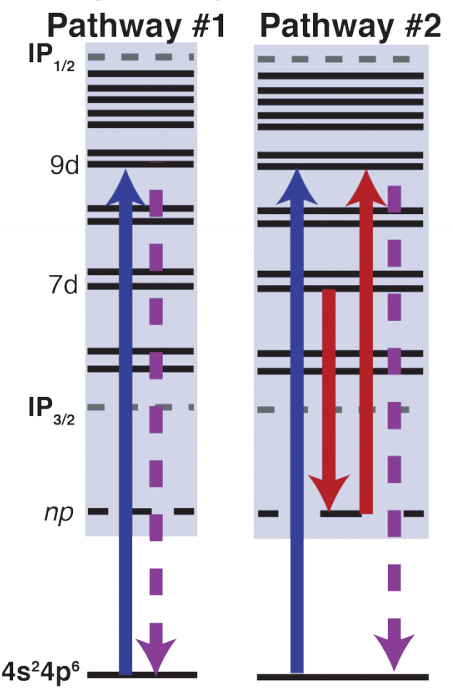

FIG 8: Pathway interference leads to distinct quantum beat oscillations in wave-mixing and transient absorption measurements. Energy level diagrams showing two pathways that interfere to generate the observed signals at (a) late delays in the four-wave mixing spectrum (white circle, Fig. 3b), (b) early delays in the four-wave mixing spectrum (black circle, Fig. 5b), and (c) early delays in the transient absorption spectrum (black circle, Fig. 7b). The assignment of relevant states is to the left of each pair of energy level diagrams. The blue, red, and dotted purple arrows indicate photons of XUV, NIR, or generated XUV wave-mixing light, respectively. The blue box illustrates the bandwidth of the XUV pulse. 
(a)

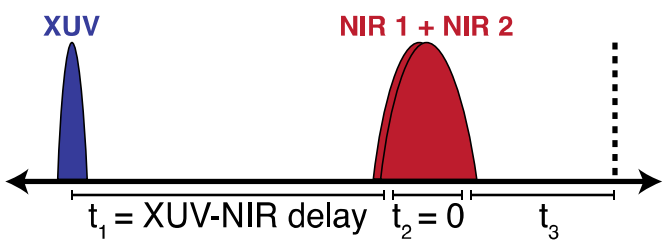

(b)

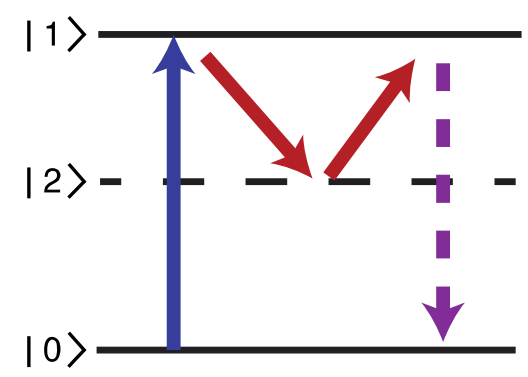

FIG 9: Pulse sequence and generalized four-wave mixing pathway. (a) The pulse sequence utilized in this experiment. (b) A non-specific wave-mixing pathway with two degenerate NIR photons. The blue, red, and dotted purple arrows indicate photons of XUV, NIR, or generated XUV wave-mixing light, respectively. See Supplementary Note 3 for more information. 


\title{
Autoionization Dynamics of $\left({ }^{2} \mathbf{P}_{1 / 2}\right) n s / d$ States in Krypton Probed by Noncollinear Wave Mixing with Attosecond Extreme Ultraviolet and Few-Cycle Near Infrared Pulses
}

\author{
Ashley P. Fidler ${ }^{1,2}$, Hugo J. B. Marroux ${ }^{1,2}$, Erika R. Warrick ${ }^{1,2}$, Etienne Bloch ${ }^{1}$, Wei Cao ${ }^{1,2}$, \\ Stephen R. Leone, ${ }^{1,2,3}$ and Daniel M. Neumark ${ }^{1,2}$ \\ ${ }^{I}$ Chemical Sciences Division, Lawrence Berkeley National Laboratory, Berkeley, CA 94720, USA \\ ${ }^{2}$ Department of Chemistry, University of California, Berkeley, CA 94720 USA \\ ${ }^{3}$ Department of Physics, University of California, Berkeley, CA 94720 USA
}

The autoionization dynamics of the $\left({ }^{2} \mathrm{P}_{1 / 2}\right)$ ns/d Rydberg states in krypton are investigated using spatially-isolated wave-mixing signals generated with a short train of subfemtosecond XUV pulses and noncollinear, few-cycle near infrared (NIR) pulses. Despite ubiquitous quantum beat oscillations from the XUV-induced coherences within the excited-state manifold, these wavemixing spectra allow for the simultaneous evaluation of autoionization lifetimes from a series of Rydberg states above the first ionization potential. Experimentally measured lifetimes of $22 \pm 8$ fs, $33 \pm 6 \mathrm{fs}$, and $49 \pm 6$ fs for the wave-mixing signals emitting from the $\left({ }^{2} \mathrm{P}_{1 / 2}\right) 6 \mathrm{~d} / 8 \mathrm{~s}$, $\left({ }^{2} \mathrm{P}_{1 / 2}\right) 7 \mathrm{~d} / 9 \mathrm{~s}$, and $\left({ }^{2} \mathrm{P}_{1 / 2}\right) 8 \mathrm{~d} / 10 \mathrm{~s}$ resonances compare favorably with lifetimes for the $\left({ }^{2} \mathrm{P}_{1 / 2}\right) 6 \mathrm{~d}, 7 \mathrm{~d}$, and $8 \mathrm{~d}$ Rydberg states determined from spectral linewidths. Analysis of the quantum beats reveals that the enhancement of wave-mixing pathways that couple the $\left({ }^{2} \mathrm{P}_{1 / 2}\right) n d$ states to themselves leads to individual reporter state-dependent decays in the wave-mixing signals. The results demonstrate the promise of wave-mixing spectroscopies with subfemtosecond XUV pulses to provide valuable insights into processes governed by electronic dynamics.

\section{Introduction}

Dynamics governed by electronic processes occur on some of the shortest timescales in nature, ranging from hundreds of attoseconds $\left(1 \mathrm{as}=10^{-18} \mathrm{~s}\right)$ to a few femtoseconds $\left(1 \mathrm{fs}=10^{-15} \mathrm{~s}\right) .^{1-3}$ Until recently, the lifetimes of these ultrafast processes typically have been determined through spectral lineshape measurements in the frequency domain. However, effects such as finite spectral resolution, heterogenous and homogeneous broadening, and spectral congestion often prevent the retrieval of accurate lifetimes associated with the underlying electronic dynamics. ${ }^{4}$ With the advent of high harmonic generation ( $\mathrm{HHG}$ )-based techniques to produce attosecond pulses in the extreme ultraviolet (XUV) and soft x-ray regimes, these dynamics have become accessible to direct measurement in the time domain. Transient absorption and photoelectron techniques utilizing attosecond pulses have been developed and employed to measure dynamic processes including autoionization, ${ }^{4,5}$ Auger decay, ${ }^{6}$ and charge migration. ${ }^{7}$ However, the observed time-dependent spectra are often complicated by overlapping spectral effects, precluding quantitative measurements in complex systems. Here, nonlinear wave-mixing spectroscopy is utilized to investigate autoionization in krypton without the interference of many of these complicating effects.

Nonlinear spectroscopies are regularly employed in the optical, infrared, and radiofrequency regimes to probe complex systems with high selectivity. ${ }^{8,9}$ In these investigations, intense electromagnetic fields coherently induce a macroscopic polarization in a target medium with significant contributions from higher-order terms, which can be expressed as:

$$
P=\varepsilon_{0}\left(\chi^{(1)} E+\chi^{(2)} E E+\chi^{(3)} E E E+\cdots\right)
$$


where $\varepsilon_{0}$ is the vacuum permittivity, $\chi^{(n)}$ is the $n^{\text {th }}$-order susceptibility, and $E$ is the electric field of the excitation. In an isotropic medium, these higher-order terms result in emission from nonlinear, or wave-mixing, processes dependent upon odd orders of $\chi^{(n)}$. Nonlinear techniques capitalize on the conservation of momentum and energy in the generation of the newly emitted field to resolve and measure signals that are often obscured in other techniques. Examples include measurements of energy transfer dynamics in light harvesting complexes with twodimensional electronic spectroscopy, ${ }^{10-12}$ studies probing the properties of surfaces and interfaces with second harmonic generation (SHG)-based methods, ${ }^{13,14}$ and imaging in biological samples with coherent anti-stokes Raman microscopy. ${ }^{15,16}$

In our laboratory, we have developed a nonlinear XUV spectroscopy utilizing attosecond XUV pulses produced by HHG and two few-cycle NIR pulses to probe electronic dynamics in atoms and small molecules. The implementation of a noncollinear beam geometry between the three pulses has proven particularly powerful, as it facilitates the spatial separation of desired wavemixing signals through phase-matching. These experiments have successfully measured both electronic and vibrational wave packet dynamics, ${ }^{17-21}$ characterized the outer well of a previously inaccessible dark state in $\mathrm{N}_{2},{ }^{20}$ and produced the first experimental demonstration of two dimensional spectroscopy in the XUV. ${ }^{21}$ In a recent study, the nonperturbative nature of noncollinear wave-mixing with these ultrashort, intense pulses was explored in He, revealing that the accumulation of the AC Stark phase over the NIR pulse duration leads to delays in higherorder signal generation. ${ }^{22}$ However, all of these experiments were performed on systems containing only long-lived Rydberg states, and the generation and time dependence of nonlinear signals from XUV-excited states that decay on ultrafast timescales has yet to be established.

In this work, multiple orders of spatially-isolated wave-mixing signals from bound and autoionizing states are generated in krypton using a noncollinear geometry between a short train of subfemtosecond XUV pulses and two time-coincident few-cycle NIR pulses. The resulting wave-mixing spectra probe the autoionization dynamics of an extended manifold of Rydberg states simultaneously. Pronounced quantum beating provides detailed information about the evolving XUV-induced coherence and NIR-mediated coupling of Rydberg states. Despite extensive coupling within the manifold of states, lifetimes of individual $4 \mathrm{~s}^{2} 4 \mathrm{p}^{5}\left({ }^{2} \mathrm{P}_{1 / 2}\right) n d$ autoionizing states can be retrieved directly in the time domain, yielding values very close to those calculated from frequency-domain linewidth measurements. Fourier analysis reveals dominant contributions from wave-mixing pathways that preferentially couple the $\left({ }^{2} \mathrm{P}_{1 / 2}\right)$ nd autoionizing states with themselves, leading to the observed reporter-state specific decays. While the mechanism is still unknown, the relative enhancement of these pathways does not appear to arise from a purely perturbative picture of wave-mixing, suggesting that nonperturbative effects may impact nonlinear signal evolution in this system. These results demonstrate that wavemixing spectroscopies constitute a powerful method for the study of ultrafast dynamics in the XUV regime, providing an alternative to spectrally complex transient absorption measurements.

\section{Experimental Methods}

The apparatus utilized for background-free attosecond wave-mixing measurements has been described previously ${ }^{20,22}$ and is shown in Fig. 1a. A $2 \mathrm{~mJ}$ per pulse, $1 \mathrm{kHz}$ repetition rate Ti:sapphire commercial femtosecond laser system (Femtopower HE, Femtolasers) produces 22 
fs NIR pulses at a central wavelength of $760 \mathrm{~nm}$. The output spectrum is spectrally broadened in a $400 \mu \mathrm{m}$ inner diameter hollow core fiber filled with 1.2 bar of neon gas and is subsequently recompressed in time by six pairs of broadband chirped mirrors (Ultrafast Innovations) and a 1 mm thick ammonium dihydrogen phosphate (ADP) plate, ${ }^{23}$ resulting in 6 fs pulses that span a wavelength range from 550 to $950 \mathrm{~nm}$. A 50:50 beamsplitter divides the broadband light into two arms. The light transmitted through the beamsplitter is focused by a $50 \mathrm{~cm}$ focal length mirror into a vacuum chamber at $10^{-3} \mathrm{~Pa}\left(10^{-6}\right.$ Torr $)$ that contains a cell statically filled with xenon gas for high harmonic generation. The interaction of the few-cycle NIR pulse with the gas generates a pulse train consisting of $2-3$ subfemtosecond XUV pulses. A $0.1 \mu \mathrm{m}$ In foil (Lebow) attenuates the driving NIR beam and selects harmonics between 11 and $17 \mathrm{eV}$, allowing for excitation of states both below and above the first ionization potential (IP) of $\mathrm{Kr}$ at $14.0 \mathrm{eV}$. A gold-coated toroidal mirror focuses the XUV through an annular mirror into a $1 \mathrm{~mm}$ pathlength (diameter) sample cell containing $1467 \mathrm{~Pa}$ (11 Torr) of krypton gas. Holes approximately 500 $\mu \mathrm{m}$ in diameter drilled through both sides of the HHG and sample cells allow for the passage of light and continuous escape of the used gas. The XUV intensity is estimated to be $10^{8}-10^{10} \mathrm{~W}$ $\mathrm{cm}^{-2}$ in the target cell due to the low conversion efficiency of HHG.

The intensity necessary to support nonlinear processes is provided by the NIR light reflected by the 50:50 beamsplitter. These few-cycle NIR pulses are delayed relative to the XUV with a piezoelectric stage (P-622 with E509 controller, Physik Instrumente (PI)) and then split into two separate arms by a 50:50 beamsplitter. Two $1 \mathrm{~m}$ focal length mirrors direct the reflected and transmitted arms vertically above and below the hole of the annular mirror in the vacuum chamber before achieving beam diameters of $\sim 100 \mu \mathrm{m}$ (FWHM) and a combined intensity of $2 \times 10^{12} \mathrm{~W} \mathrm{~cm}^{-2}$ in the target cell. To ensure spatial separation of signals originating from multiphoton wave-mixing pathways from the harmonics, the two NIR beams intersect the XUV in a noncollinear geometry at angles of approximately $18 \mathrm{mrad}\left(1^{\circ}\right)$ and $13 \mathrm{mrad}\left(0.75^{\circ}\right)$ for the upper and lower arms, respectively (Fig. 1b). In the experiments described here, the two NIR pulses are time-coincident. A second piezoelectric stage is placed in the arm transmitted through the 50:50 beamsplitter to compensate for daily fluctuations in the length of the two arms.

Temporal and spatial overlap between the XUV and both NIR arms is determined by removing the metallic foil blocking the copropagating NIR after HHG and inserting a removable mirror into the beam path after the annular mirror, directing all three NIR beams outside the vacuum apparatus to an optical CMOS camera (DCC1545M, Thorlabs). The positions of the stages are modified manually until interference fringes are observed between all beams (Supplementary Fig.1). The crossing angle $\theta_{\text {crossing }}$ can be calculated from the fringe spacing via Eqn. (2):

$$
2 \sin ^{-1}\left(\lambda_{N I R} / 2 x\right)
$$$$
\theta_{\text {crossing }}=
$$

where $\lambda_{N I R}$ is the central NIR wavelength and $x$ is the fringe spacing.

A $0.15 \mu \mathrm{m}$ In foil (Lebow) attenuates the NIR beams after the sample cell. The transmitted XUV light is dispersed in frequency by a gold-coated flat-field grating (01-0464, Hitachi) and recorded as a function of both frequency and phase-matching divergence angle by a $1340 \times 400$ pixel xray CCD camera (Pixis XO 400B, Princeton Instruments). From the absorption features of longlived Rydberg states in argon, the spectral resolution at $14.3 \mathrm{eV}$ is expected to be $19 \mathrm{meV}$. Signal 
from a total of 1500 laser pulses is accumulated at each XUV-NIR delay. Here, positive delays indicate that the XUV pulse precedes the time coincident NIR pulses (Fig. 1c). A step size of 300 as was chosen to resolve short-lived features around time overlap. To examine longer-lived features, the step size was increased to $1 \mathrm{fs}$ and $3 \mathrm{fs}$ at XUV-NIR delays of $130 \mathrm{fs}$ and $312 \mathrm{fs}$, respectively. The energy axis of the CCD camera was calibrated daily using atomic transition line data available from the National Institute of Standards and Technology (NIST) for the Rydberg states of argon. Except where noted, the data are presented in terms of absorbance:

$$
\mathrm{A}=-\log _{10}\left(I / I_{0}\right)
$$

where $I$ is the light transmitted through the krypton sample at a given XUV-NIR delay and $I_{0}$ is a reference spectrum taken without krypton gas in the sample cell before each delay-dependent scan. Absorption features have positive absorbance values while emission features from wavemixing processes have negative absorbance values. For wave-mixing signals spatially isolated from the incident XUV pulse, $I_{0}$ comprises the dark counts from the CCD plus any residual scattered light from the XUV and NIR arms. This signal presentation mode is chosen to emphasize the weaker wave-mixing signals and indicate that they originate explicitly from newly generated light.

\section{Results}

\section{A. Spatial Isolation of Resonance-Enhanced Wave-Mixing Emission Signals}

A noncollinear geometry between a short XUV pulse train and two few-cycle NIR pulses results in background-free wave-mixing signals in krypton from the $4 \mathrm{~s}^{2} 4 \mathrm{p}^{5}\left({ }^{2} \mathrm{P}_{1 / 2}\right) n l$ states above the first IP $(14.0 \mathrm{eV})$. These states can autoionize to form the $\mathrm{Kr}^{+}{ }^{2} \mathrm{P}_{3 / 2}$ state. To generate wave-mixing signals that probe these autoionizing states, the broadband XUV pulse train (13.5 to $16.0 \mathrm{eV})$ induces a coherent superposition between the ground state and a manifold of bound and autoionizing states that decays with the dephasing time (polarization lifetime) of the constituent states. ${ }^{24}$ The addition of two moderately intense, noncollinear NIR pulses produces wave-mixing signals that emit at a wavevector dependent upon the sum of the initial XUV $\left(k_{X U V}\right)$ and NIR $\left(k_{N I R} 1, k_{N I R} 2\right)$ wavevectors.

An X-ray CCD camera image taken at temporal overlap of all three pulses is shown in Figure 2a. The image comprises several bands, each of which results from one or more phase-matching conditions illustrated in Fig. 2b. The spatial dimension of the image is reported in divergence angle relative to the incident XUV beam. For each photon of the wave-mixing pathway resonant with an electronic transition in the atom, the intensity of the emitted signal is enhanced. All onephoton dipole-forbidden $n p$ (dark) states accessible by one NIR photon from the $n s / d$ autoionizing states are located below the first IP.

In the band centered at $0 \mathrm{mrad}$, positive absorbance values indicate the linear absorption of high harmonic XUV light by atomic resonances both above and below the first IP. At time overlap, the XUV-induced polarization is perturbed by the NIR pulses, leading to a variety of spectral effects, including spectral broadening and modification of absorption lineshapes, shifting of atomic resonances, and depletion of features ${ }^{2,4,25,26}$ As shown by the phase-matching diagrams in Figure $2 \mathrm{~b}$, the spectrum at $0 \mathrm{mrad}$ will also exhibit contributions from multiphoton wave-mixing pathways that terminate without an overall change of the emission angle such that $k_{X U V}-$ 


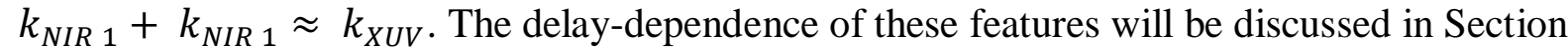
IIIC.

A distinct band of strong negative absorbance (emission) features is observed below the harmonic axis from -2.5 to $-4.3 \mathrm{mrad}$ at the bright state energies. These features originate from wave-mixing pathways that employ photons from both NIR arms (NIR 1 and NIR 2). As shown in Fig. $2 \mathrm{~b}$, both third $\left(k_{4 W M}=k_{X U V}-k_{N I R 1}+k_{N I R 2}\right)$ and fifth $\left(k_{6 W M}=k_{X U V}-k_{N I R 1}+\right.$

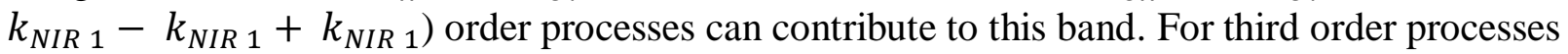
dependent on both NIR pulses, wave-mixing signals emit at divergence angles, $\phi_{\text {div }}$, approximately equal to the following expression:

$$
\phi_{d i v} \approx \frac{v_{N I R 1} \theta_{N I R 1}+v_{N I R 2} \theta_{N I R 2}}{v_{X U V}}
$$

where $v_{N I R 1}$ and $v_{N I R 2}$ are the frequencies of the two relevant NIR interactions and $v_{X U V}$ is the frequency of the initial XUV excitation. For example, as shown in Fig. 2b, one of the wavemixing pathways that gives rise to the signal at $14.26 \mathrm{eV}$ involves one XUV photon from the ground state to the $\left({ }^{2} \mathrm{P}_{1 / 2}\right) 9 \mathrm{~s}$ bright state $(14.26 \mathrm{eV})$ and two NIR photons resonant with the $\left({ }^{2} \mathrm{P}_{1 / 2}\right) 6 \mathrm{p}$ dark state $(1.5 \mathrm{eV})$. Using the measured NIR crossing angles of 18 and $13 \mathrm{mrad}$ and Eqn. (4), this pathway emits at a divergence angle of $-3.3 \mathrm{mrad}$, which is consistent with the experimentally observed central divergence angle of $-3.4 \mathrm{mrad}$. Discrepancies between observed and calculated emission angles can be attributed to error in the measured crossing angles and the presence of multiple different wave-mixing pathways that emit at slightly different angles. In the transient grating picture of wave-mixing, these signals are described as the first diffraction order. ${ }^{27,28}$ For simplicity, because third order signals should be more intense than fifth order signals, we refer to this band of features as the four-wave mixing signal.

A weaker band of wave-mixing signals can be observed between -6.1 and -7.8 mrad. As shown in Fig. $2 b$, these features primarily correspond to fifth order wave-mixing processes that employ

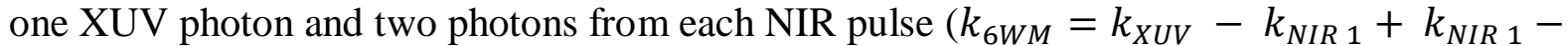
$\left.k_{N I R} 1+k_{N I R} 1\right)$. The divergence angle expected for these processes can be calculated using a relationship similar to Eqn. (4):

$$
\phi_{d i v} \approx \frac{2\left(v_{N I R 1} \theta_{N I R 1}+v_{N I R 2} \theta_{N I R 2}\right)}{v_{X U V}}
$$

For a five photon process involving one $14.26 \mathrm{eV}$ photon and four $1.5 \mathrm{eV}$ photons, the calculated divergence angle is $-6.6 \mathrm{mrad}$. This band of fifth order features is referred to as the six-wave mixing signal.

\section{B. Delay-Dependent Wave-Mixing Emission from Autoionizing States in Krypton}

The temporal profile of the bound and autoionizing states probed can be accessed by integrating over the angular range of the four-wave mixing signals on the CCD camera image (Fig. 2a, middle band). Plotting the result as function of the delay between the XUV pulse train and the two time-coincident NIR pulses reveals information about the evolution of the XUV-induced coherence. Fig. 3a shows a false color plot of the four-wave mixing signal as a function of photon energy and XUV-NIR delay. Wave-mixing signals appear as negative absorbance (emission) features at the energies of atomic resonances. High-resolution photoabsorption linewidth measurements previously determined the lifetimes of the first $d$ - and $s$-character states above the IP, $\left({ }^{2} \mathrm{P}_{1 / 2}\right) 6 \mathrm{~d}$ and $\left({ }^{2} \mathrm{P}_{1 / 2}\right) 8 \mathrm{~s}$, to be 23.5 fs and 949.5 fs respectively, indicating that the $d$ - 
character states are better coupled to the underlying continuum. ${ }^{29}$ As expected from examining the overlap integral, the lifetimes of autoionizing states lengthen with principal quantum number, $n$, and therefore with increasing energy. The spectral resolution is insufficient to fully resolve individual wave-mixing signals emitting from adjacent $n d / n s$ states as well as from the finely spaced states approaching the IPs.

Just below the first IP at $14.0 \mathrm{eV}$, wave-mixing features emitting from the bound states do not decay appreciably within the timeframe of the experiment. In contrast, wave-mixing signals emitting from the spectrally overlapped long-lived $n s$ and short-lived $n d$ autoionizing states above the IP exhibit visible decays. At each resonance, an intense, relatively short-lived feature can be distinguished from weaker, longer-lived quantum beat oscillations that persist for the entirety of the experiment. This short-lived feature is approximately five times more intense than the longer-lived component. Notably, the duration of the shorter-lived component increases with the principal quantum number of the emission state, following the known trend for autoionizing state lifetimes. The quantum beat oscillations that underlie both the short and long time components in the time-domain spectrum arise from the interference of wave-mixing pathways, as described in more detail below. Constituent pathways can be identified from the oscillation frequencies as determined by a Fourier transform along the delay axis (Fig. 3b). Strong, distinct features appear at $0.067 \mathrm{eV}, 0.105 \mathrm{eV}, 0.176 \mathrm{eV}$, and $0.28 \mathrm{eV}$ in the lower-lying states.

To assess the short-lived time component, three wave-mixing features at the energies of $14.1 \mathrm{eV}$, $14.26 \mathrm{eV}$, and $14.37 \mathrm{eV}$ are fit to a convolution of the instrumental response and a biexponential decay using a Levenberg-Marquardt least squares algorithm (Fig. 4). Given that the long-lived quantum beats endure well over an order of magnitude longer than the short-lived features, the long-time component was fixed at an arbitrarily long value. Varying the value of the long time constant between $500 \mathrm{fs}$ and $1 \mathrm{~ns}$ did not significantly alter the value of the short-time constant. This fitting procedure yields decays of $22 \pm 8 \mathrm{fs}, 33 \pm 6 \mathrm{fs}$, and $49 \pm 6 \mathrm{fs}$ for the short-lived components of the features emitting from the $\left({ }^{2} \mathrm{P}_{1 / 2}\right) 6 \mathrm{~d} / 8 \mathrm{~s},\left({ }^{2} \mathrm{P}_{1 / 2}\right) 7 \mathrm{~d} / 9 \mathrm{~s}$, and $\left({ }^{2} \mathrm{P}_{1 / 2}\right) 8 \mathrm{~d} / 10 \mathrm{~s}$ states respectively, verifying the qualitative observation that the duration of the short-lived component increases with the principal quantum number of the reporter state.

Windowing the four-wave mixing spectrum to include only the first 130 fs after time overlap emphasizes the dynamics of the short-lived component (Fig. 5a). In the dense band of emission approaching the second ionization potential at $14.63 \mathrm{eV}$, the frequency of the dominant quantum beat increases with energy, creating hyperbolic band structures. As shown in Fig. 5b, the Fourier transform of just these early delays $(-20 \mathrm{fs}-130 \mathrm{fs})$ is visibly distinct from the transform that encompasses longer delays (Fig. 3b). The origin of these differences is covered in Section IVA. The majority of features in Fig. 5b fall on lines with unity slope and an intercept at the energy of one of the lower lying $\left({ }^{2} \mathrm{P}_{1 / 2}\right)$ nd states. As in the full transform, these features must originate from the interference of two multiphoton pathways in the wave-mixing spectrum.

Higher order six-wave mixing signals are captured by integrating between -6.1 and -7.8 mrad on the CCD camera image in Fig. 2a. A false color plot demonstrating the evolution of the six-wave emission as a function of photon energy and XUV-NIR delay is shown in Fig. 6a. Although the signal-to-noise ratio is lower, the isolated fifth order wave-mixing features exhibit dynamics equivalent to those observed for previously described four-wave mixing signals. However, six- 
wave mixing emission emerges at later XUV-NIR delays than four-wave mixing emission. As shown in Fig. 6b, the six-wave mixing signal emitting from the $\left({ }^{2} \mathrm{P}_{1 / 2}\right) 6 \mathrm{~d} / 8 \mathrm{~s}$ autoionizing state is delayed by $1.8 \pm 0.4$ fs relative to the corresponding four-wave mixing signal. A similar delay was seen in $\mathrm{He}^{22}$ and is discussed further in Section IVB.

\section{Comparison to Transient Absorption of Autoionizing States in Krypton}

Integrating over the absorption band situated between 2 and $-1.2 \mathrm{mrad}$ on the CCD camera image yields an attosecond transient absorption measurement of the autoionizing state dynamics of $\mathrm{Kr}$ (Fig. 7a). Here, positive (absorption) features arise at the energies of the autoionizing states. Substantial energy shifts of the resonances around pulse overlap and adjacent bands at later delays indicated in ellipses $i$ and ii can be attributed to the AC Stark effect, which gives rise to a laser-imposed phase shift that accumulates over the NIR pulse duration..$^{22,25,26,30,31}$ In addition to the AC Stark effects, evidence of wave-mixing processes can be observed at early delays through quantum beat oscillations highlighted in ellipse iii and described in detail in previous publications. ${ }^{25}$ As in the wave-mixing spectra, the quantum beats approaching the second IP increase in frequency with photon energy, creating hyperbolic band structures. The most prominent features in the corresponding Fourier transform appear along lines of unity slope that intercept the $y$-axis at the energies of bright state resonances (Fig. 7b). The delay dependence extending to nearly $1 \mathrm{ps}$ and the corresponding Fourier transform are provided as Supplementary Fig. 2.

\section{Discussion}

\section{A. Explanation of Experimentally Observed Wave-Mixing Signals}

The results of these noncollinear wave-mixing experiments with subfemtosecond XUV and fewcycle NIR pulses conclusively indicate that nonlinear XUV spectroscopies can access ultrafast decays of electronic excited states with notable advantages over transient absorption. From the CCD camera image alone (Fig. 2a), it is clear that the pulses employed in these experiments supply sufficient intensity to induce wave-mixing from the $4 \mathrm{~s}^{2} 4 \mathrm{p}^{5}\left({ }^{2} \mathrm{P}_{3 / 2}\right) n l$ and $\left({ }^{2} \mathrm{P}_{1 / 2}\right) n l$ states of $\mathrm{Kr}$, which exhibit a larger range of photoabsorption cross sections and decay rates than previously studied systems. Particularly notable is the observation of spatially isolated wavemixing signals at the energies of the spectrally-broad XUV-generated autoionizing states above the first IP. The time and frequency-domain spectra of these emission signals reveal a global view of autoionization dynamics occurring above the first IP in this system (Fig. 3a).

Despite substantial quantum beat oscillations indicative of contributions from multiple different states to each emission signal, the duration of the intense, short-lived component of the wavemixing features increases with the principal quantum number of the reporter (emitting) state, following the trend expected for the Rydberg state lifetimes. As shown in Table I, the experimentally measured decay constants for the short-lived components of the $\left({ }^{2} \mathrm{P}_{1 / 2}\right) 8 \mathrm{~s} / 6 \mathrm{~d}$, $9 \mathrm{~s} / 7 \mathrm{~d}$ and $10 \mathrm{~s} / 8 \mathrm{~d}$ wave-mixing features agree favorably with the population lifetimes of the $\left({ }^{2} \mathrm{P}_{1 / 2}\right) 6 \mathrm{~d}, 7 \mathrm{~d}$ and $8 \mathrm{~d}$ Rydberg states ${ }^{29}$. While more disruptive quantum beat oscillations preclude quantitative fits of higher photon energy features, the lifetimes retrieved for these lower energy emission signals suggest that the short-lived time component can be attributed to wave-mixing 
pathways probing the $\left({ }^{2} \mathrm{P}_{1 / 2}\right)$ nd autoionizing states. The relationship between the measured wavemixing emission lifetime and that of the $\left({ }^{2} \mathrm{P}_{1 / 2}\right)$ nd reporter state is noteworthy as it indicates that a single wave-mixing pathway dominates over other pathways that also contribute to the emission signal.

Fourier analysis of the wave-mixing emission signals can provide insight into the wave-mixing pathways that contribute to the delay dependence of the emitted signals. The Fourier transform of the time-domain data including XUV-NIR delays up to $965 \mathrm{fs}$ largely provides evidence of coherences from the long-lived $n s$ autoionizing states with lifetimes on the scale of picoseconds (Fig. 3b). For example, the prominent $0.176 \mathrm{eV}$ frequency peak obtained from the $\left({ }^{2} \mathrm{P}_{1 / 2}\right) 8 \mathrm{~s}$ emission signal at $14.10 \mathrm{eV}$ (circled in Fig. 3b) can be assigned to the simultaneous XUV excitation of the $8 \mathrm{~s}(14.10 \mathrm{eV})$ and $9 \mathrm{~s}(14.27 \mathrm{eV})$ autoionizing states. The XUV-induced coherence of these two states is coupled by two NIR photons to the $\left({ }^{2} \mathrm{P}_{1 / 2}\right) 8$ s reporter state via a dark state of $p$ - or $f$-electronic character (Fig. 8a). The broad bandwidth of both the XUV and NIR pulses allows not only for the XUV-induced coherent excitation of a manifold of excited states, but also for extensive dark-state mediated coupling of states within in the $\sim 0.5 \mathrm{eV} \mathrm{NIR}$ bandwidth. Other prominent Fourier peaks at low photon energies can be assigned to the XUVinduced coherence of the $10 \mathrm{~s}$ and $11 \mathrm{~s}$ states $(0.067 \mathrm{eV}), 9 \mathrm{~s}$ and $10 \mathrm{~s}$ states $(0.105 \mathrm{eV})$, and $8 \mathrm{~s}$ and $10 \mathrm{~s}$ states $(0.28 \mathrm{eV})$. These features demonstrate the extensive coupling between Rydberg states that occurs within this system, emphasizing that the observed wave-mixing signals probe the evolution of multiple XUV-excited states.

Fourier analysis of only early delays ( $130 \mathrm{fs})$ reveals contributions from the shorter-lived $n d$ autoionizing states (Fig. 5b). The hyperbolic band structures in Fig. 5a generate peaks in the photon-energy resolved Fourier transform that fall on lines with unity slope, which are highlighted by white lines in Fig. 5b. All features along the white lines arise from wave-mixing pathways that couple the state at the intercept with the final state at which the feature is observed. Here, the lines intercept the y-axis at the energies of the $n d$ states preferentially. To produce such lines, the second interfering pathway is best described by a wave-mixing process with two degenerate NIR photons that couples the reporter state with itself. An example of two interfering pathways that lead to a peak in the early-delay Fourier transform are provided as Pathways \#1 and \#2 in Fig. 8b. Pathways with two degenerate NIR photons like Pathway \#2 appear to dominate the total emitted signal from the manifold of autoionizing states at early times, leading to the hyperbolic band structures and the intense, short-lived component observed in the time-domain wave-mixing signals, as will be discussed further in Section VB.

Moreover, because of large contributions from these NIR-degenerate pathways, the delaydependent wave-mixing spectra provide more insight into the underlying excited state dynamics than a traditional transient absorption measurement. In previous attosecond transient absorption studies, AC-Stark effects were shown to limit the accuracy of lifetime measurements to only lineouts taken exactly on-resonance. ${ }^{4}$ As shown in Fig. 7a, the severity of the AC Stark shift of the resonances around pulse overlap precludes any lineout measurement of the excited state lifetime, particularly of the short-lived $\left({ }^{2} \mathrm{P}_{1 / 2}\right) n d$ states. Without resolvable decays, the disappearance of quantum beat oscillations provides the most convincing evidence of autoionization in the transient absorption spectrum. The quantum beats produce peaks that appear along unity slope lines in the Fourier transform reminiscent of the features observed in 
the transform of the noncollinear wave-mixing signals at early delays (Fig. 7b). These lines are interpreted as the interference of a one-photon, linear response pathway (Fig. 8c, Pathway \#1) and a three photon, wave-mixing pathway (Fig 8c, Pathway \#2) that emit at the state in which the beat occurs. The interference of one and three photon pathways is discussed extensively in the transient absorption literature. ${ }^{25}$

\section{B. General Characteristics of Wave Mixing with Ultrashort Pulses}

The conspicuous differences between transient absorption and wave-mixing spectra can be attributed to the presence or absence of the XUV-excitation field. In transient absorption experiments, NIR-induced features, including those generated by the AC Stark effect and wavemixing pathways, interfere with the incident high harmonic XUV light. The XUV field thus acts as a reference field, allowing for phase-sensitive heterodyne detection ${ }^{9}$. Using a square law detector, the intensity of the detected signal can be described by the following relationship:

$$
I_{\text {signal }}=\left|E_{X U V}+E_{E m i t}\right|^{2}=\left|E_{X U V}\right|^{2}+\left|E_{E m i t}\right|^{2}+2 \Re\left(E_{E m i t} E_{X U V}^{*}\right)
$$

where $I_{\text {signal }}$ is the intensity of the detected signal, $E_{X U V}$ is the electric field of the incident XUV pulse train, and $E_{E m i t}$ is the electric field of generated polarization signal. When the incident XUV field is present, $E_{\text {Emit }}$ includes both the first order polarization and any higher-order polarization fields. In a perturbative picture, the intensity of the incident XUV field can be assumed to be greater than the generated light. The intensity detected by the CCD camera can then be described by the following expression:

$$
I_{\text {signal }}=\left|E_{X U V}+E_{E m i t}\right|^{2} \approx\left|E_{X U V}\right|^{2}+2 \Re\left(E_{\text {Emit }} E_{X U V}^{*}\right)
$$

The time-domain dynamics of the detected field in this self-heterodyned detection scheme are dominated by the cross-term. ${ }^{9}$

For wave-mixing measurements in a noncollinear geometry, the reference XUV field does not overlap spatially with generated wave-mixing signals, resulting in a homodyne detection scheme. In optical homodyne detection, the square modulus of the incident field and the cross term can be ignored. The detected intensity can therefore be adequately described by only the square modulus of the emitted field, which only includes wave-mixing fields and is proportional the polarization response function, $R^{(3)}\left(t_{3}, t_{2}, t_{1}\right)$ :

$$
\left|E_{E m i t}\right|^{2}=\left|E_{W M}\right|^{2} \propto\left|R^{(3)}\left(t_{3}, t_{2}, t_{1}\right)\right|^{2}
$$

where $E_{W M}$ is the electric field of the wave-mixing signals. The time convention for $t_{1}, t_{2}$, and $t_{3}$ is defined in Fig. 9a. The relevant components of the wave mixing response function for a single, arbitrary four-wave mixing pathway (Fig. 9b) utilized in our experiment can be defined as:

$$
R^{(3)}\left(t_{3}, t_{2}, t_{1}\right) \propto \mu_{01}^{2} \mu_{12}^{2} e^{-i\left(\omega_{1}-\omega_{0}\right) t_{1}-t_{1} / T_{2}}
$$

where $\mu_{01}$ and $\mu_{12}$ are the transition dipoles associated with the transition between states $|0\rangle$ and $|1\rangle$ and between states $|1\rangle$ and $|2\rangle$. The frequencies of states $|0\rangle$ and $|1\rangle$ are described by $\omega_{0}$ and $\omega_{1}$, respectively. The decay term $T_{2}$ describes the dephasing time, which equals twice the population lifetime $\left(T_{1}\right)$ for a system in which dephasing due to the environment is negligible ${ }^{9}$. A more detailed derivation of the third-order response function using diagrammatic perturbation theory is provided as Supplementary Note 3. In heterodyne detection schemes dominated by the cross-term, the observed decay is equal to $T_{2}$. To demonstrate the impact of this homodyne 
detection scheme on a time-domain signal, the response function is incorporated into the Eqn. (8) as follows:

$$
\left|E_{W M}\right|^{2} \propto\left|\mu_{01}^{2} \mu_{12}^{2} e^{-i\left(\omega_{1}-\omega_{0}\right) t_{1}-t_{1} / T_{2}}\right|^{2} \propto \mu_{01}^{4} \mu_{12}^{4} e^{-2^{t_{1}} / T_{2}}
$$

The time-integrated response of the detector over $t_{3}$ is not detailed here. Note that, without contributions from the cross-term, frequency-dependent terms that result in AC Stark hyperbolic side bands are eliminated by the square modulus. Furthermore, in this homodyne geometry, the measured decay equals the excited state lifetime as $T_{1}=T_{2} / 2$.

The above treatment applies for the emission of a single wave-mixing pathway. However, because of the broad bandwidths of the pulses employed in these experiments, the time evolution of individual emission signals reflects the contributions of multiple different wave-mixing pathways. ${ }^{21}$ Pathways like those in Fig. 8a-b interfere, resulting in quantum beat oscillations, and should collectively dictate the decay of the emission feature. As demonstrated by the perturbative simulation shown in Supplementary Note 3, significant contributions from multiple pathways with nondegenerate NIR photons, like Pathway \#1 in Fig. 8b, effectively eliminate lifetime variations between wave-mixing signals emitting from different reporter states. However, the experimental wave-mixing spectrum does exhibit reporter-state dependent lifetime variations. The duration of the short-lived component visibly increases with the principal quantum number of the reporter state. Moreover, the measured lifetimes track those of the Rydberg states coupled to the reporter states by a NIR-degenerate pathway like Pathway \#2 in Fig. 8b. Supported by the early-delay Fourier transform (Fig. 5b), these NIR-degenerate pathways appear to be systematically enhanced relative to other pathways for the entire manifold of autoionizing states, allowing for the preferential coupling of the reporter states to themselves. The enhancement of NIR-degenerate pathways therefore leads to reporter-state dependent variations in the delaydependence of these wave-mixing signals.

While not yet fully characterized in this system, the enhancement of NIR-degenerate pathways may potentially be explained by effects that cannot be fully captured by a perturbative picture of wave mixing. In a perturbative picture for a fully-resonant wave-mixing process, the interaction of each pulse with the medium is treated as the linear absorption or emission of a photon from an eigenstate of the atom. For the system studied here, the emission of a broadband $(\sim 0.5 \mathrm{eV}) \mathrm{NIR}$ photon projects the XUV-induced coherence onto dark states located below the IP. The simultaneous interaction of a second NIR photon generates resonance-enhanced wave-mixing signals that emit from all states within the bandwidth of the NIR pulse, including the autoionizing states above the first IP. However, features suggesting significant deviations from a perturbative wave-mixing regime can be identified in this system. Previous experiments on longlived Rydberg and dressed states in atomic helium have demonstrated that phase gratings due to the nonresonant AC Stark effect are instrumental in the formation of wave-mixing signals in this experimental geometry, leading to delays in emergence of higher diffraction orders. ${ }^{22}$ As shown in Fig. 6b, the six-wave mixing signal emitting at the energy of the $\left({ }^{2} \mathrm{P}_{1 / 2}\right) 6 \mathrm{~d} / 8$ s autoionizing state is delayed by $1.8 \pm 0.4$ fs relative to the four-wave mixing signal, indicating that a purely perturbative wave mixing picture cannot adequately capture all the features observed of this experiment. 
In previous literature, the AC-Stark effect has been implicated in nonperturbative wave-mixing regimes as well as in pathway enhancement ${ }^{32-35}$ and therefore may provide an attractive mechanism by which to explain the enhancement of NIR-degenerate pathways in this experiment. However, the development of theoretical models that provide a general understanding of the impact of nonperturbative effects on wave-mixing signals is currently an active area of research. ${ }^{34,36-39}$ As few nonperturbative treatments of multilevel systems are currently available, more work is necessary to clarify the role of the AC-Stark effect on the evolution of wave-mixing signals in a complex system like the one studied here. Regardless of the mechanism, this experiment suggests that the enhancement of desirable wave-mixing pathways can assist in the measurement of ultrafast lifetimes by, in essence, reducing the complexity of the wave-mixing experiments. Once fully characterized, the mechanism that allows for pathway enhancement, such as the AC-Stark effect, may ultimately be utilized as a tool to enhance or eliminate particular nonlinear processes, providing experimentalists with substantive control over signal evolution.

\section{Conclusions}

Utilizing a noncollinear geometry with a short train of subfemtosecond XUV pulses and two few-cycle NIR pulses, we directly probe the time-domain evolution of spatially isolated wavemixing signals from autoionizing states that decay on a variety of different timescales. This work not only represents the first successful generation of isolated XUV wave-mixing signals from states that decay on these timescales, but also demonstrates that wave-mixing experiments performed in a noncollinear geometry may confer significant advantages over transient absorption experiments when measuring ultrafast decays. Due to the suppression of AC Stark energy level shifting and sidebands in homodyne wave-mixing experiments without the high harmonic XUV field, lifetimes of the short-lived $\left({ }^{2} \mathrm{P}_{1 / 2}\right)$ nd autoionizing states can be extracted directly from the time-domain signal despite persistent quantum beat oscillations. The observed lifetime trend can be attributed to wave-mixing pathways coupling the reporter states to themselves that dominate at the early delays. While the origins of this pathway enhancement are not yet fully established, delays between the emergence of four and six-wave mixing emission signals due to the accumulation of an AC-Stark grating provide evidence that nonperturbative effects impact signal generation and may influence signal evolution in this system. This work suggests that the selection of desired pathways, either through pathway enhancement as shown here or via multidimensional spectroscopic techniques, can support the measurement of ultrafast lifetimes, revealing dynamics that would have otherwise been obscured by the multiplexed nonlinear response of the system.

\section{Acknowledgements}

This work was supported by the Director, Office of Science, Office of Basic Energy Sciences through the Atomic, Molecular, and Optical Sciences Program of the Division of Chemical Sciences, Geosciences, and Biosciences of the US Department of Energy at LBNL under contract no. DE-AC02-05CH11231. A.P.F. acknowledges funding from the National Science Foundation Graduate Research Fellowship Program.

\section{Supplementary Material}


See supplementary material for CMOS camera images for pulse overlap determination, a transient absorption spectrum of the $\left({ }^{2} \mathrm{P}_{1 / 2}\right) n s / d$ states over nearly a picosecond of delays, and a fully resonant, perturbative simulation of the wave-mixing spectrum.

\section{References}

${ }^{1}$ F. Krausz and M. Ivanov, Rev. Mod. Phys. 81, 163 (2009).

2 A.R. Beck, D.M. Neumark, and S.R. Leone, Chem. Phys. Lett. 624, 119 (2015).

${ }^{3}$ K. Ramasesha, S.R. Leone, and D.M. Neumark, Annu. Rev. Phys. Chem. 67, 41 (2016).

${ }^{4}$ B. Bernhardt, A.R. Beck, X. Li, E.R. Warrick, M.J. Bell, D.J. Haxton, C.W. McCurdy, D.M. Neumark, and S.R. Leone, Phys. Rev. A - At. Mol. Opt. Phys. 89, 1 (2014).

${ }^{5}$ X. Li, B. Bernhardt, A.R. Beck, E.R. Warrick, A.N. Pfeiffer, M. Justine Bell, D.J. Haxton, C. William McCurdy, D.M. Neumark, and S.R. Leone, J. Phys. B At. Mol. Opt. Phys. 48, (2015).

${ }^{6}$ M. Drescher, M. Hentschel, R. Kienberger, M. Uiberacker, V. Yakovlev, a Scrinzi, T.

Westerwalbesloh, U. Kleineberg, U. Heinzmann, and F. Krausz, Nature 419, 803 (2002).

${ }^{7}$ F. Calegari, A. Trabattoni, A. Palacios, D. Ayuso, M.C. Castrovilli, J.B. Greenwood, P.

Decleva, F. Martín, and M. Nisoli, J. Phys. B At. Mol. Opt. Phys. 49, 142001 (2016).

${ }^{8}$ S. Mukamel, Principles of Nonlinear Optical Spectroscopy (Oxford University Press, New York, 1995).

${ }^{9}$ P. Hamm and M.T. Zanni, Concepts and Methods of 2D Infrared Spectroscopy (Cambridge University Press, Cambridge, 2011).

${ }^{10}$ T.R. Calhoun, N.S. Ginsberg, G.S. Schlau-Cohen, Y.C. Cheng, M. Ballottari, R. Bassi, and G.R. Fleming, J. Phys. Chem. B 113, 16291 (2009).

${ }^{11}$ G.S. Schlau-Cohen, T.R. Calhoun, N.S. Ginsberg, E.L. Read, M. Ballottari, R. Bassi, R. van Grondelle, and G.R. Fleming, J. Phys. Chem. B 113, 15352 (2009).

${ }^{12}$ D. Zigmantas, E.L. Read, T. Mancal, T. Brixner, A.T. Gardiner, R.J. Cogdell, and G.R. Fleming, Proc. Natl. Acad. Sci. 103, 12672 (2006).

${ }^{13}$ Y.R. Chen, Nature 337, 519 (1989).

${ }^{14}$ P.E. Ohno, S.A. Saslow, H.F. Wang, F.M. Geiger, and K.B. Eisenthal, Nat. Commun. 7, 13587 | (2016).

${ }^{15}$ C.L. Evans and X.S. Xie, Annu. Rev. Anal. Chem. 1, 883 (2008).

${ }^{16}$ A. Zumbusch, G.R. Holtom, and X.S. Xie, Phys. Rev. Lett. 82, 4142 (1999).

${ }^{17}$ W. Cao, E.R. Warrick, A. Fidler, S.R. Leone, and D.M. Neumark, Phys. Rev. A 94, 021802 (2016).

${ }^{18}$ W. Cao, E.R. Warrick, A. Fidler, D.M. Neumark, and S.R. Leone, Phys. Rev. A - At. Mol. Opt. Phys. 94, 053846 (2016).

${ }^{19}$ W. Cao, E.R. Warrick, A. Fidler, S.R. Leone, and D.M. Neumark, Phys. Rev. A 97, 023401 (2018).

${ }^{20}$ E.R. Warrick, A. Fidler, W. Cao, E. Bloch, D.M. Neumark, and S.R. Leone, Faraday Discuss. 212, 157 (2018).

${ }^{21}$ H.J.B. Marroux, A.P. Fidler, D.M. Neumark, and S.R. Leone, Sci. Adv. 4, (2018).

${ }^{22}$ A.P. Fidler, S.J. Camp, E.R. Warrick, E. Bloch, H.J.B. Marroux, D.M. Neumark, K.J. Schafer, M.B. Gaarde, and S.R. Leone, Nat. Commun. 10, 1384 (2019).

${ }^{23}$ H. Timmers, Y. Kobayashi, K.F. Chang, M. Reduzzi, D.M. Neumark, and S.R. Leone, Opt. Lett. 42, 811 (2017).

${ }^{24}$ S. Bengtsson, E.W. Larsen, D. Kroon, S. Camp, M. Miranda, C.L. Arnold, A. L'Huillier, K.J. Schafer, M.B. Gaarde, L. Rippe, and J. Mauritsson, Nat. Photonics 11, 252 (2017). 
${ }^{25}$ W. Cao, E.R. Warrick, D.M. Neumark, and S.R. Leone, New J. Phys. 18, 013041 (2016).

${ }^{26}$ M. Wu, S. Chen, S. Camp, K.J. Schafer, and M.B. Gaarde, J. Phys. B At. Mol. Opt. Phys. 49, 062003 (2016).

${ }^{27}$ A. Blouin, M.-M. Denariez Roberge, and P. Galarneau, J. Opt. Soc. Am. B Opt. Phys. 8, 578 (1991).

${ }^{28}$ E.J. Brown, Q. Zhang, and M. Dantus, J. Chem. Phys. 110, 5772 (1999).

${ }^{29}$ K. Maeda, K. Ueda, and K. Ito, J. Phys. B At. Mol. Opt. Phys. 26, 1541 (1993).

${ }^{30}$ C. Ott, A. Kaldun, P. Raith, K. Meyer, M. Laux, J. Evers, C.H. Keitel, C.H. Greene, and T. Pfeifer, Science (80-. ). 340, 716 (2013).

${ }^{31}$ S. Chen, M. Wu, M.B. Gaarde, and K.J. Schafer, Phys. Rev. A - At. Mol. Opt. Phys. 88, 033409 (2013).

${ }^{32}$ A.M. Levine, N. Chencinski, W.M. Schreiber, A.N. Weiszmann, and Y. Prior, Phys. Rev. A 35, 2550 (1987).

${ }^{33}$ W. Chalupczak, W. Gawlik, and J. Zachorowski, Phys. Rev. A 49, 4895 (1994).

${ }^{34}$ D.A. Coppeta, P.L. Kelley, P.J. Harshman, and T.K. Gustafson, 53, 925 (1996).

${ }^{35}$ C.-Y. Tai, R.T. Deck, and C. Kim, Phys. Rev. A 37, 163 (1988).

${ }^{36}$ L. Chen, E. Palacino-González, M.F. Gelin, and W. Domcke, J. Chem. Phys. 147, (2017).

${ }^{37}$ M.F. Gelin, D. Egorova, and W. Domcke, J. Phys. Chem. B 115, 5648 (2011).

${ }^{38}$ V. V. Strelkov, Phys. Rev. A 93, 1 (2016).

39 J.M. Shacklette and S.T. Cundiff, J. Opt. Soc. Am. B 20, 764 (2003). 
Tables

\begin{tabular}{|c|c|c|}
\hline Reporter State & Four-Wave Mixing Lifetime (fs) & Literature Lifetime (fs) $^{29}$ \\
\hline$\left({ }^{2} \mathrm{P}_{1 / 2}\right) 6 \mathrm{~d}$ & $22 \pm 8$ & 23.5 \\
\hline$\left({ }^{2} \mathrm{P}_{1 / 2}\right) 7 \mathrm{~d}$ & $33 \pm 6$ & 34.0 \\
\hline$\left({ }^{2} \mathrm{P}_{1 / 2}\right) 8 \mathrm{~d}$ & $49 \pm 6$ & 47.1 \\
\hline
\end{tabular}

TABLE 1. Comparison of measured four-wave mixing lifetimes with literature values. Lifetimes measured for three four-wave mixing signals in the time-domain spectrum compare favorably with lifetimes of the $\left({ }^{2} \mathrm{P}_{1 / 2}\right)$ nd reporter states determined from linewidth measurements. 


\section{Figures}
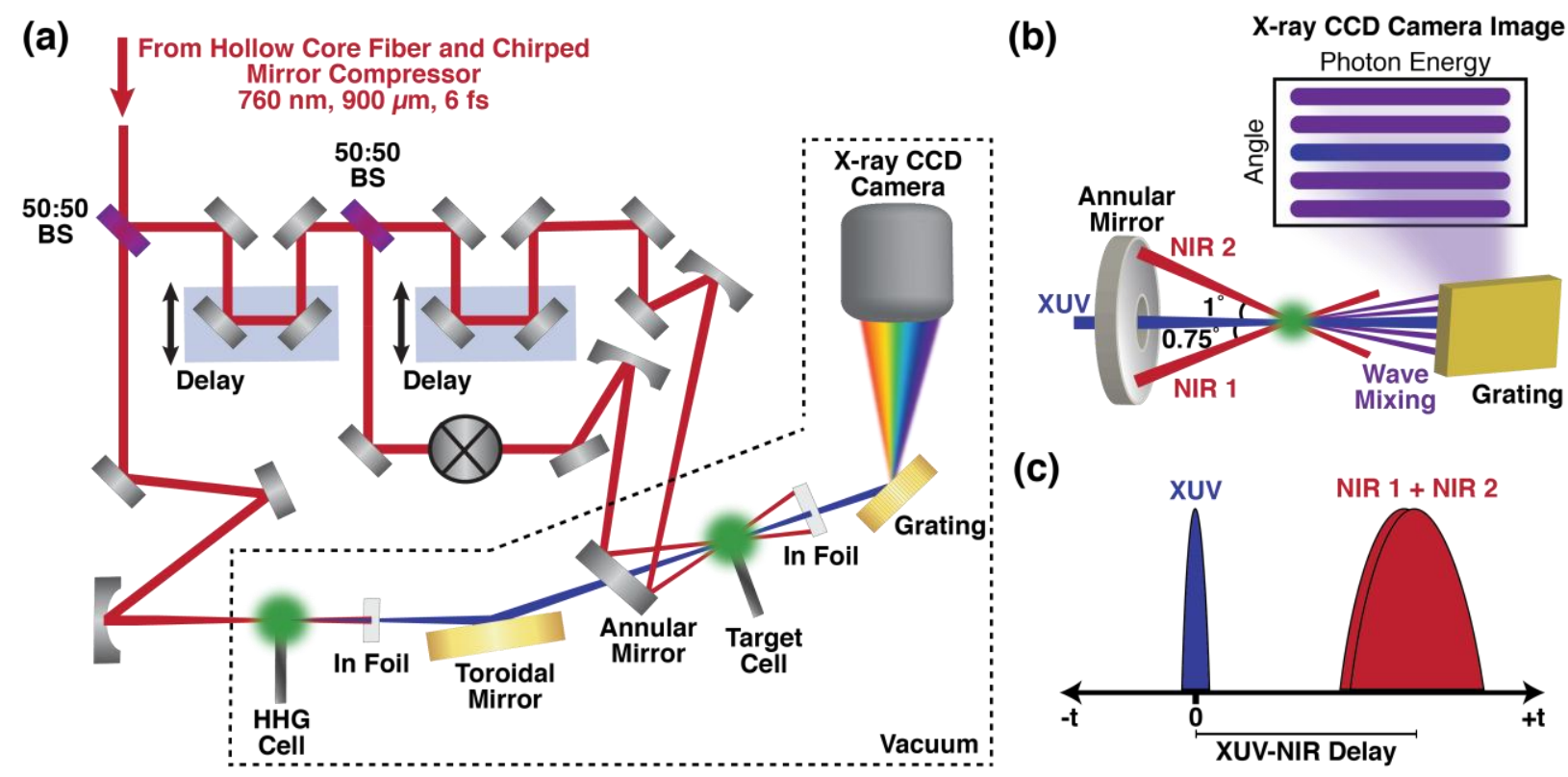

FIG 1. Experimental apparatus and pulse sequence. (a) Experimental apparatus after NIR pulse compression used for noncollinear wave-mixing experiments. (b) The interaction of the noncollinear NIR pulses with the XUV-excited sample leads to spatial separation of wavemixing signals. The NIR beam oriented below the hole mirror is labelled NIR 1 while the beam oriented above the mirror is labelled NIR 2. (c) Dynamics encoded in wave-mixing signals are measured as a function of the time delay between the XUV and time-coincident NIR pulses. Positive delays indicate that the XUV pulse precedes the NIR pulses. 

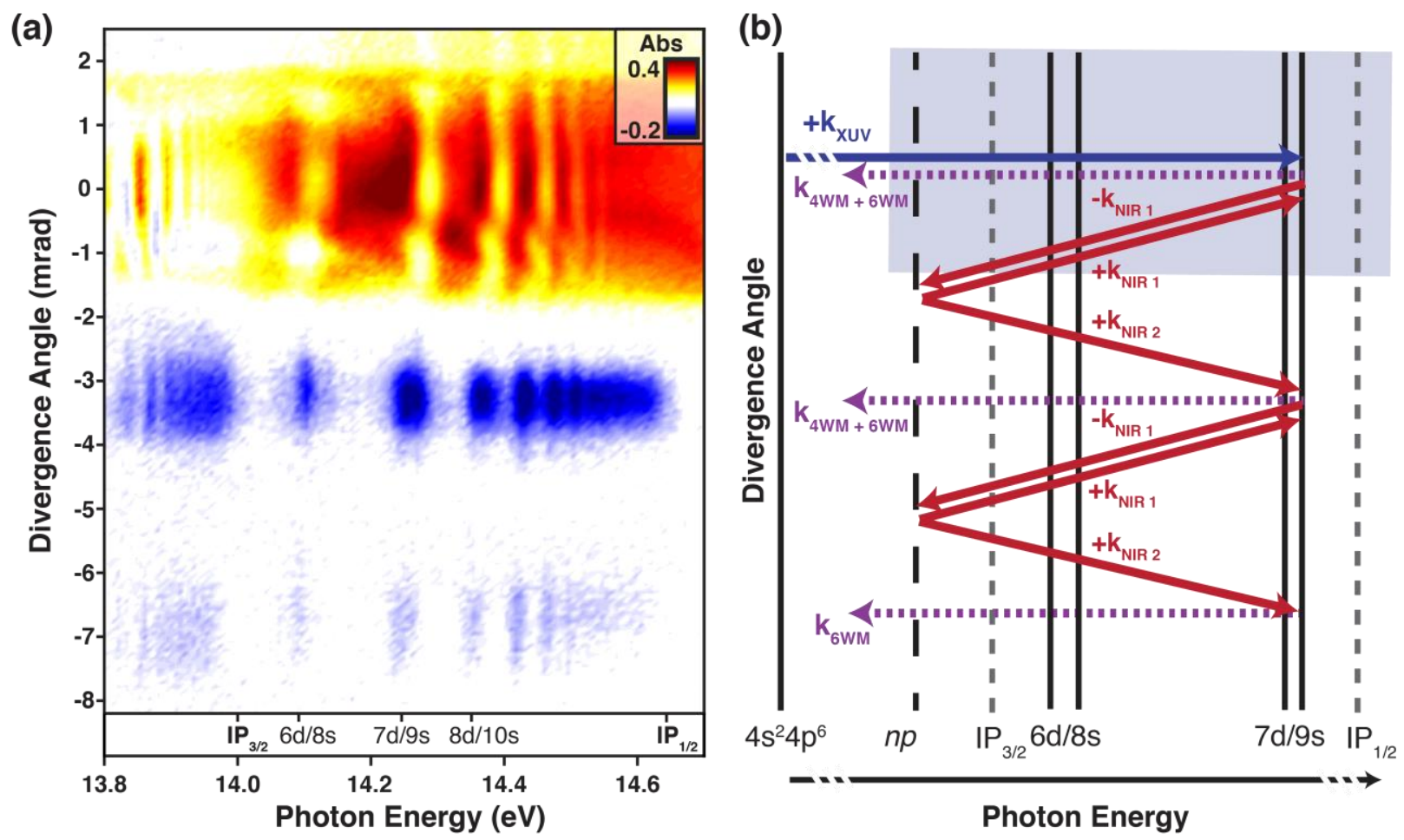

FIG 2: Spatially-dependent emission of multiple orders of wave-mixing signals. (a) An x-ray charge coupled device (CCD) image plotted as function of photon energy and phase-matching divergence angle exhibits three distinct bands of absorption or wave-mixing signals. The energies of the first two ionization potentials and several key autoionizing states are provided at the bottom of the image. (b) Example phase-matching diagrams show wave-mixing pathways that emit at each of the three bands at the $\left({ }^{2} \mathrm{P}_{1 / 2}\right) 7 \mathrm{~d} / 9 \mathrm{~s}$ resonance at $14.26 \mathrm{eV}$. The two bands closest to $0 \mathrm{mrad}$ contain contributions from multiple orders of wave-mixing signals. The blue, red, and dotted purple arrows indicate photons of XUV, NIR, or generated XUV wave-mixing light, respectively. 
(a)

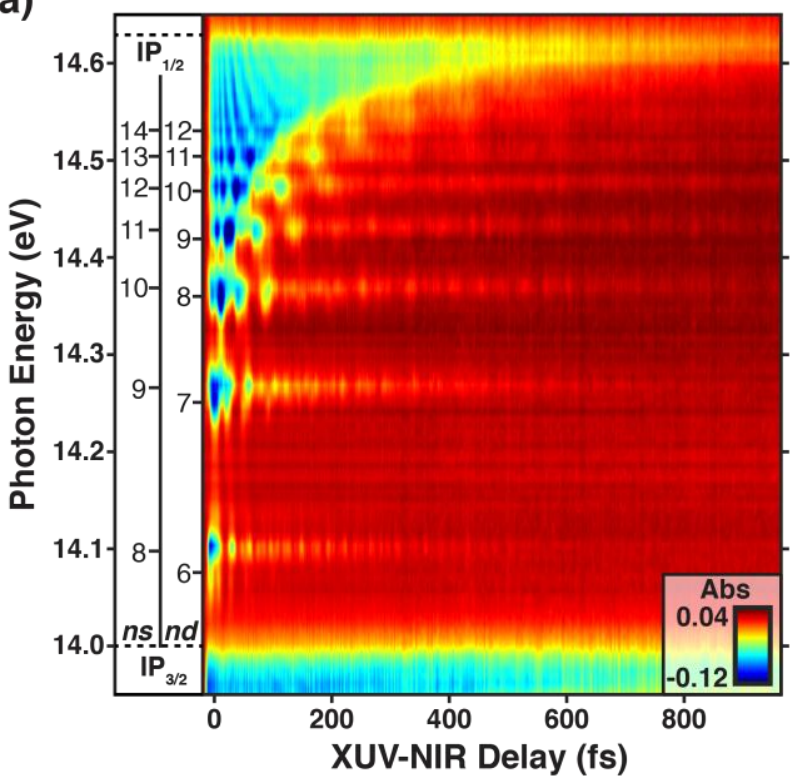

(b)

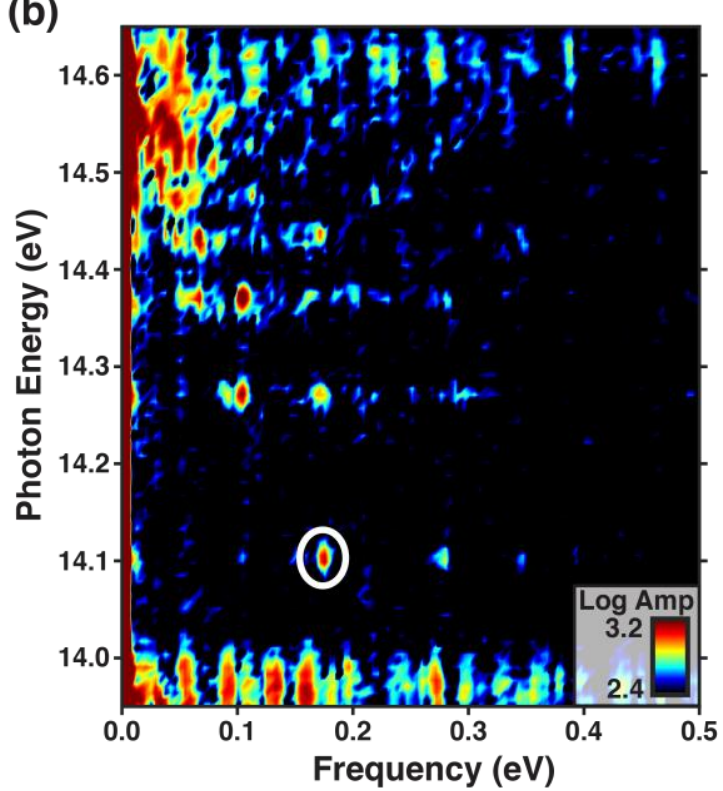

FIG 3: Four-wave mixing measurements of $\left({ }^{2} \mathbf{P}_{1 / 2}\right) n l$ autoionizing states of $K r$. (a) Emission spectrum of the four-wave mixing signals as a function of energy and XUV-NIR delay. State assignments are provided to the left of the panel. (b) Fourier analysis of the delay-dependent spectrum. The white circle highlights a feature arising from the interference of two wave-mixing pathways that will be discussed explicitly in Fig. 8 . 

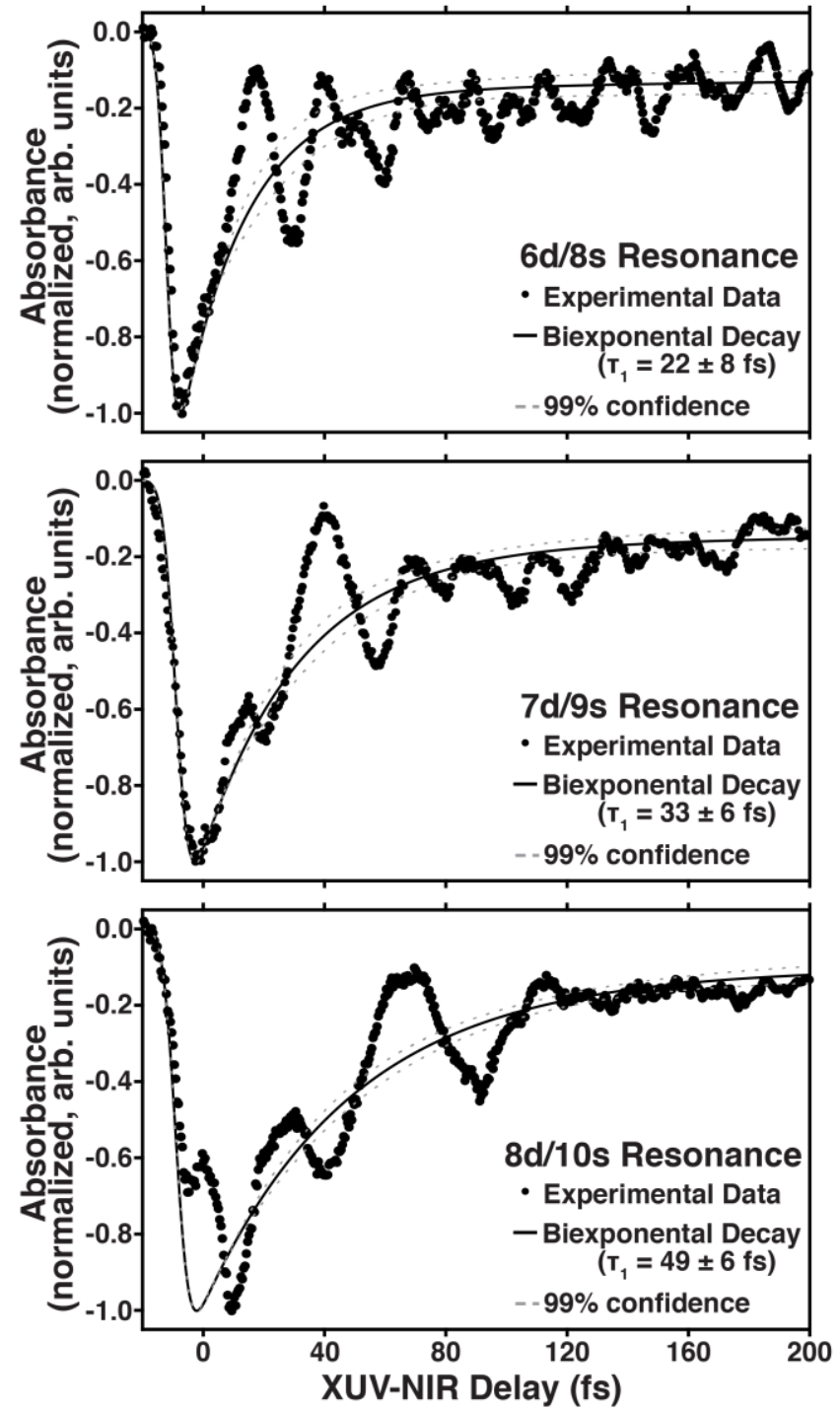

FIG 4: Lifetime analysis of ultrafast wave-mixing decays. Lineouts taken from the delaydependent four-wave mixing signal at the (a) $\left({ }^{2} \mathrm{P}_{1 / 2}\right) 6 \mathrm{~d} / 8 \mathrm{~s}$ resonance $(14.1 \mathrm{eV})$, (b) $\left({ }^{2} \mathrm{P}_{1 / 2}\right) 7 \mathrm{~d} / 9 \mathrm{~s}$ resonance $(14.26 \mathrm{eV})$, and $(\mathbf{c})\left({ }^{2} \mathrm{P}_{1 / 2}\right) 8 \mathrm{~d} / 10 \mathrm{~s}$ resonance $(14.37 \mathrm{eV})$ are fit to biexponential decays with an arbitrary long time constant. The fit short time constant compares favorably with the excited state population lifetimes known for the $\left({ }^{2} \mathrm{P}_{1 / 2}\right) 6 \mathrm{~d},\left({ }^{2} \mathrm{P}_{1 / 2}\right) 7 \mathrm{~d}$, and $\left({ }^{2} \mathrm{P}_{1 / 2}\right) 8 \mathrm{~d}$ states, respectively (See text for details). 
(a)

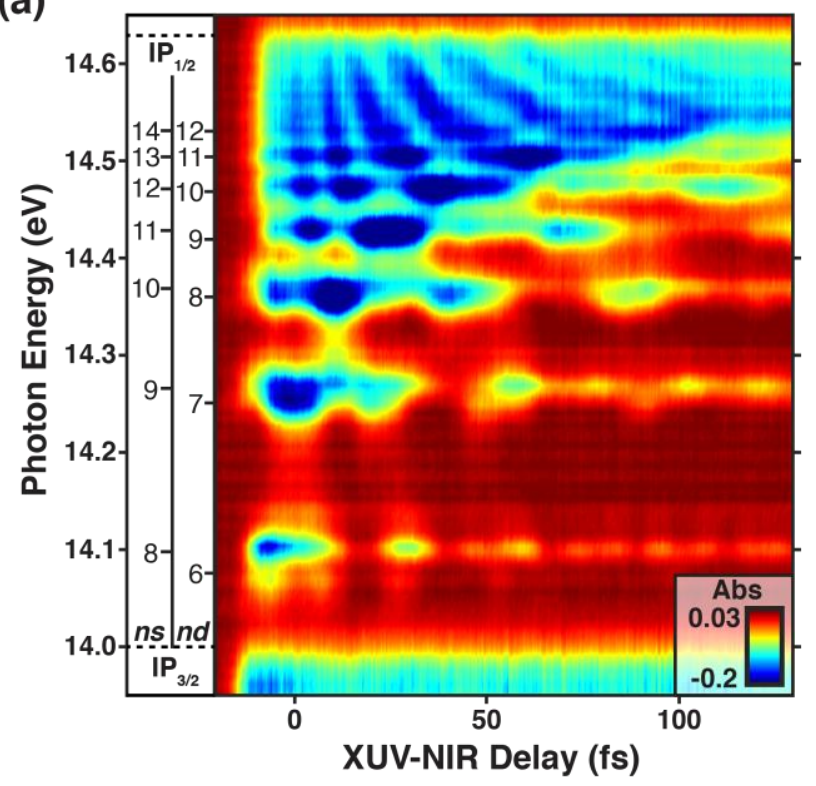

(b)

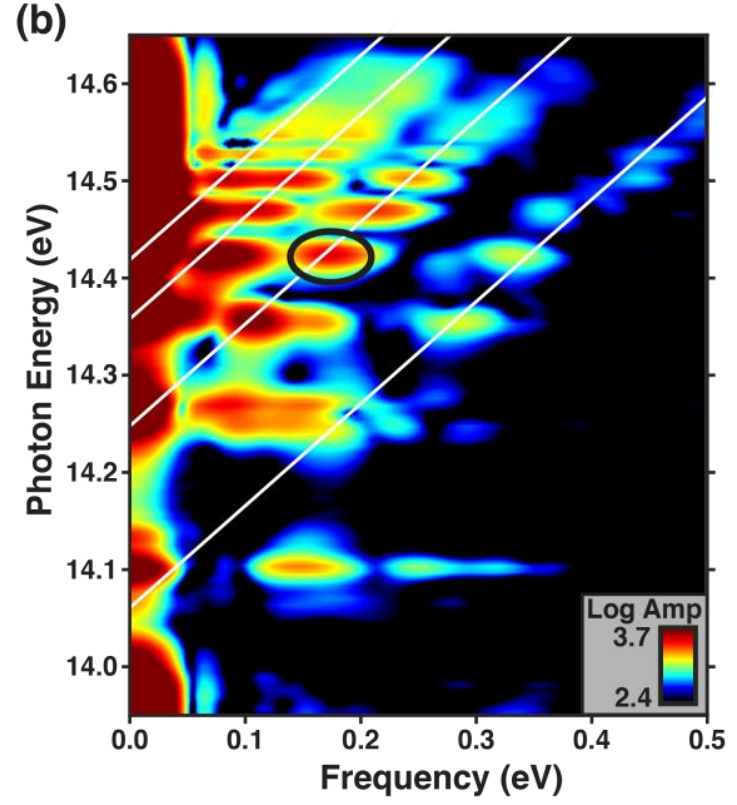

FIG 5: Early delay-dependence of four-wave mixing signals in $\mathrm{Kr}$. (a) Emission spectrum of the four-wave mixing signals windowed to encompass only XUV-NIR delays from -20 to $130 \mathrm{fs}$. State assignments are provided to the left of the panel. (b) Fourier analysis of the windowed delay-dependent spectrum. The white lines have unity slope and indicate that the features along them arise in part from a wave-mixing pathway that begins with XUV excitation to the state at the intercept. The feature highlighted by the black circle will be identified in Fig. 8b. 
(a)

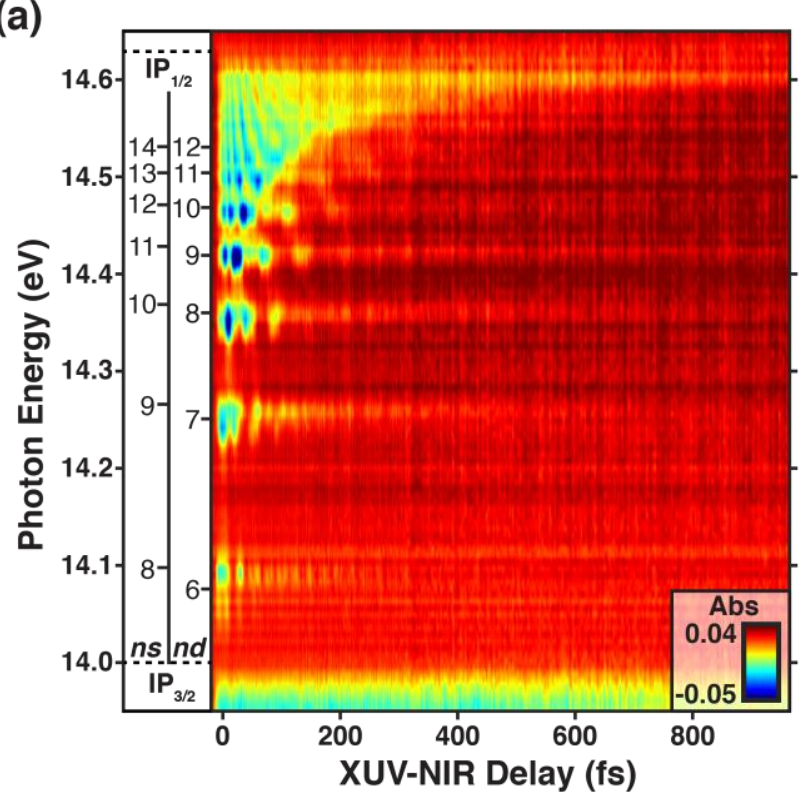

(b)

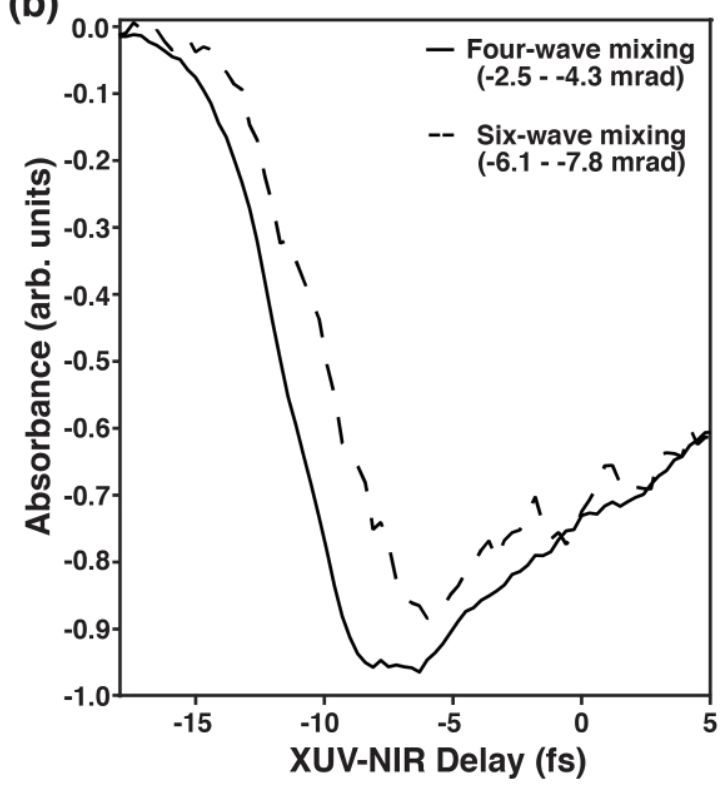

FIG 6: Six-wave mixing measurements of autoionizing states of Kr. (a) Absorption spectrum of the six-wave mixing signals as a function of energy and XUV-NIR delay. State assignments are provided to the left of the panel. (b) Lineouts of wave-mixing signals generated at the $\left({ }^{2} \mathrm{P}_{1 / 2}\right) 7 \mathrm{~d} / 9$ s resonance $(14.1 \mathrm{eV})$ obtained by integrating over the predominately four-wave mixing emission $(-2.5--4.5 \mathrm{mrad})$ or the six-wave mixing emission $(-6.1--7.8 \mathrm{mrad})$ bands in the CCD camera image shown in Fig $2 \mathrm{a}$. 
(a)

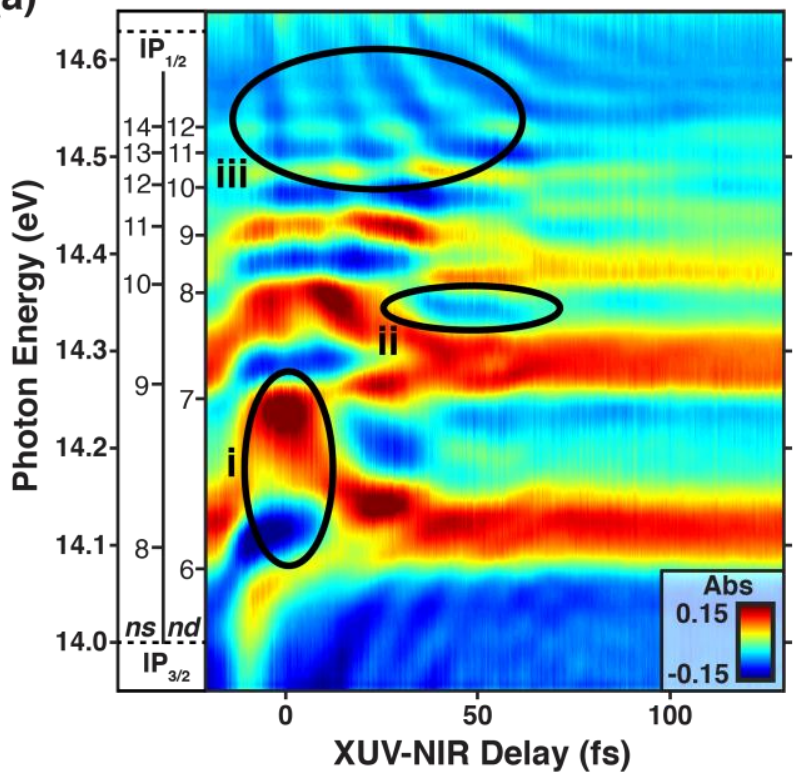

(b)

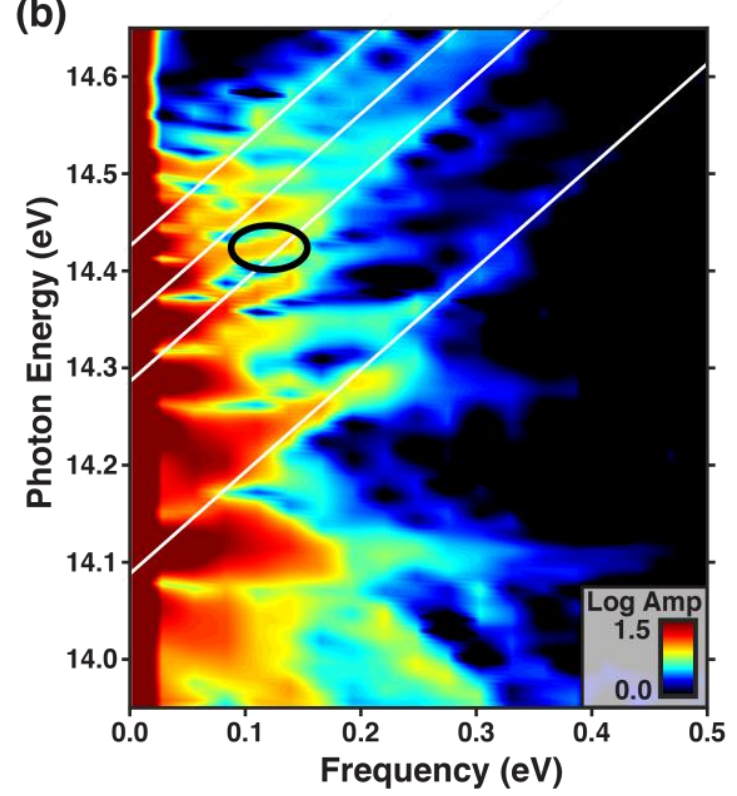

FIG 7: Transient absorption measurements of autoionizing states of $\mathbf{K r}$. (a) Absorption is plotted as a function of XUV-NIR delay and energy. States assignments are located to the left of the panel. Black ellipses indicate features of interest discussed in the text. (b) Fourier analysis of the delay-dependent spectrum. The white lines possess a unity slope and indicate that the features along them arise from the interference of the linear response and a wave-mixing pathway that begins with an XUV excitation to the state at the intercept. The feature circled in black will be discussed further in Fig. 8a. 
(a) Four-Wave Mixing Late Delays

Pathway \#1

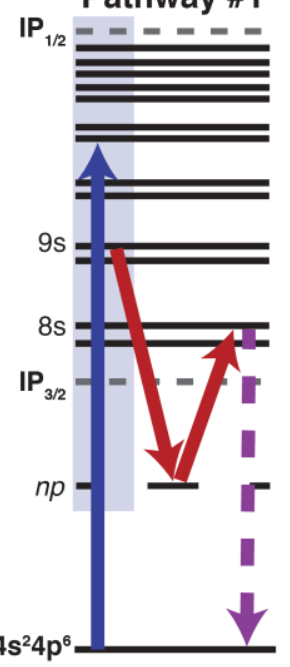

(b) Four-Wave Mixing Early Delays

Pathway \#2
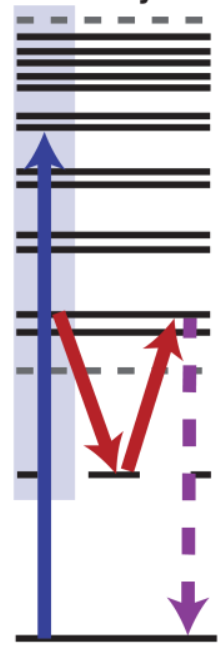

Pathway \#1

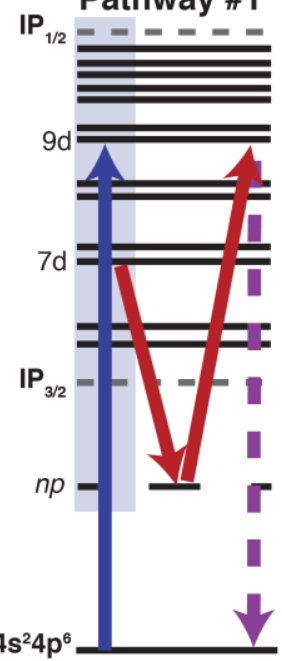

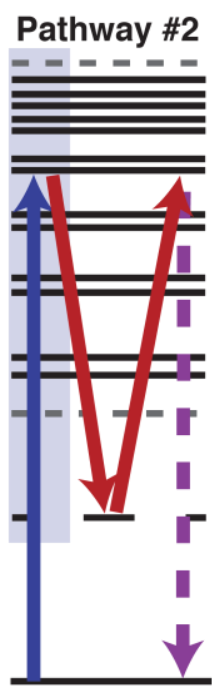

(c) Transient Absorption Early Delays

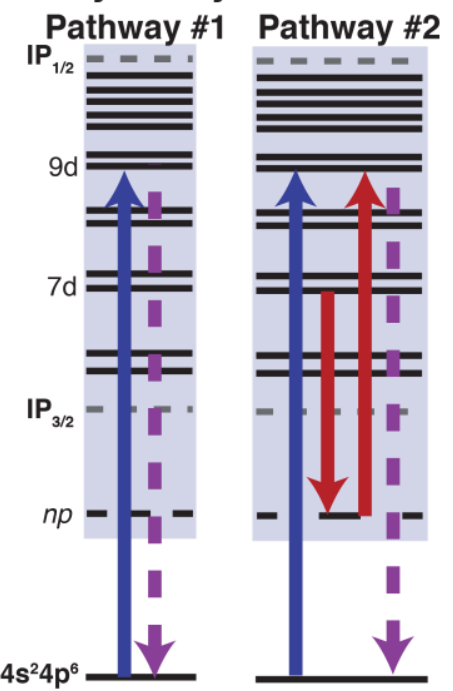

FIG 8: Pathway interference leads to distinct quantum beat oscillations in wave-mixing and transient absorption measurements. Energy level diagrams showing two pathways that interfere to generate the observed signals at (a) late delays in the four-wave mixing spectrum (white circle, Fig. 3b), (b) early delays in the four-wave mixing spectrum (black circle, Fig. 5b), and (c) early delays in the transient absorption spectrum (black circle, Fig. 7b). The assignment of relevant states is to the left of each pair of energy level diagrams. The blue, red, and dotted purple arrows indicate photons of XUV, NIR, or generated XUV wave-mixing light, respectively. The blue box illustrates the bandwidth of the XUV pulse. 
(a)

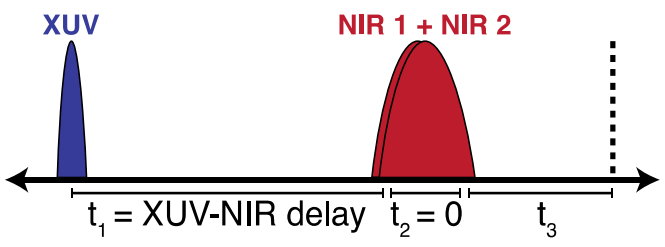

(b)

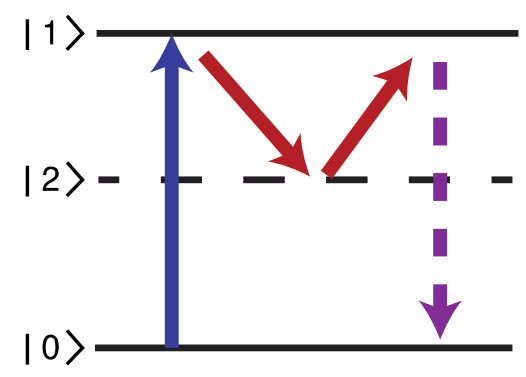

FIG 9: Pulse sequence and generalized four-wave mixing pathway. (a) The pulse sequence utilized in this experiment. (b) A non-specific wave-mixing pathway with two degenerate NIR photons. The blue, red, and dotted purple arrows indicate photons of XUV, NIR, or generated XUV wave-mixing light, respectively. See Supplementary Note 3 for more information. 


\title{
- Supplementary Information -
}

\section{Autoionization Dynamics of $\left({ }^{2} P_{1 / 2}\right) n s / d$ States in Krypton Probed by Noncollinear Wave Mixing with Attosecond Extreme Ultraviolet and Few-Cycle Near Infrared Pulses}

\author{
Ashley P. Fidler ${ }^{1,2}$, Hugo J. B. Marroux ${ }^{1,2}$, Erika R. Warrick ${ }^{1,2}$, Etienne Bloch ${ }^{1}$, Wei Cao ${ }^{1,2}$, \\ Stephen R. Leone, ${ }^{1,2,3}$ and Daniel M. Neumark ${ }^{1,2}$ \\ ${ }^{1}$ Chemical Sciences Division, Lawrence Berkeley National Laboratory, Berkeley, CA 94720, USA \\ ${ }^{2}$ Department of Chemistry, University of California, Berkeley, CA 94720 USA \\ ${ }^{3}$ Department of Physics, University of California, Berkeley, CA 94720 USA
}




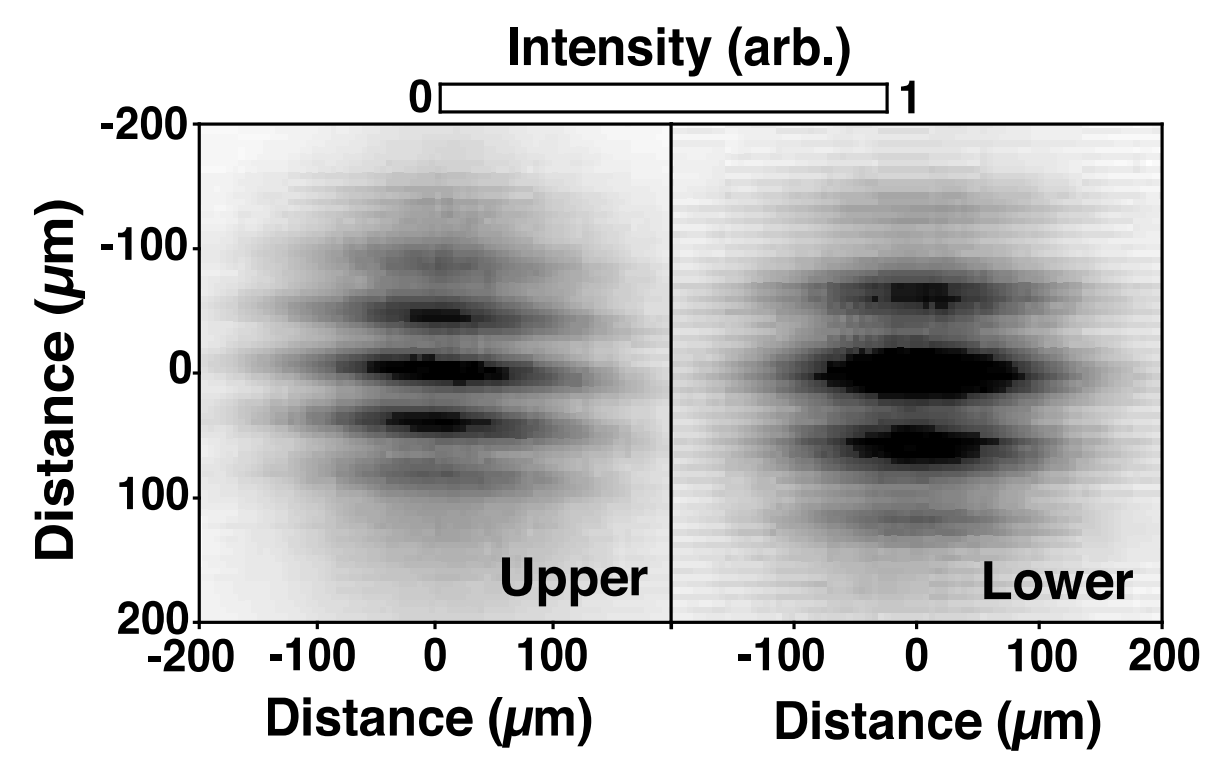

Supplementary Fig. 1: Fringes on CMOS camera images identify crossing angles. Overlap fringes formed from the interference of the noncollinear (a) upper and (b) lower NIR arms with the NIR pulse that drives HHG.

(a)

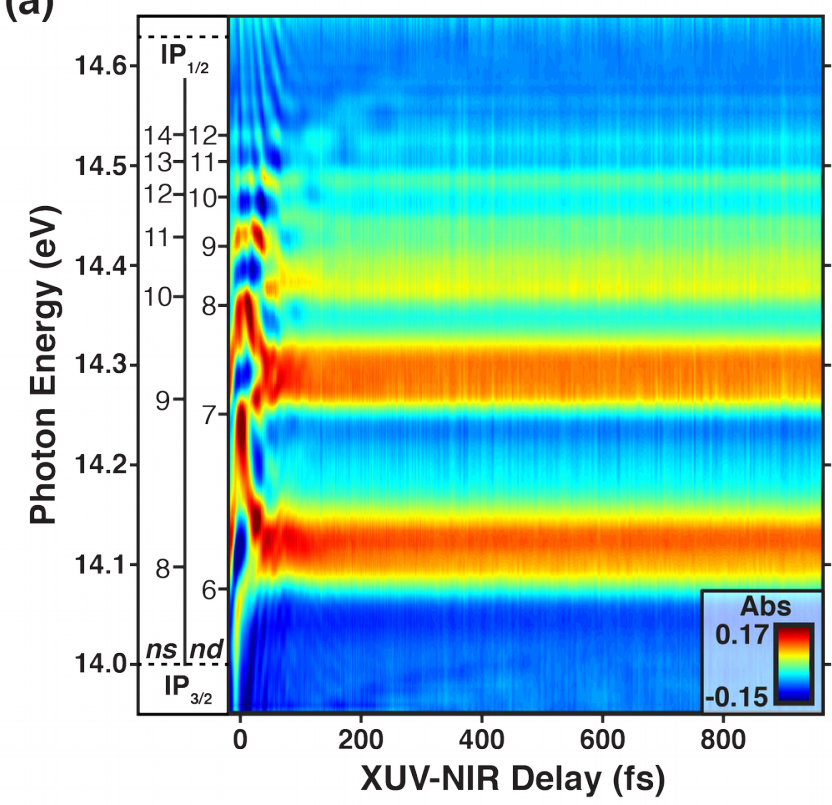

(b)

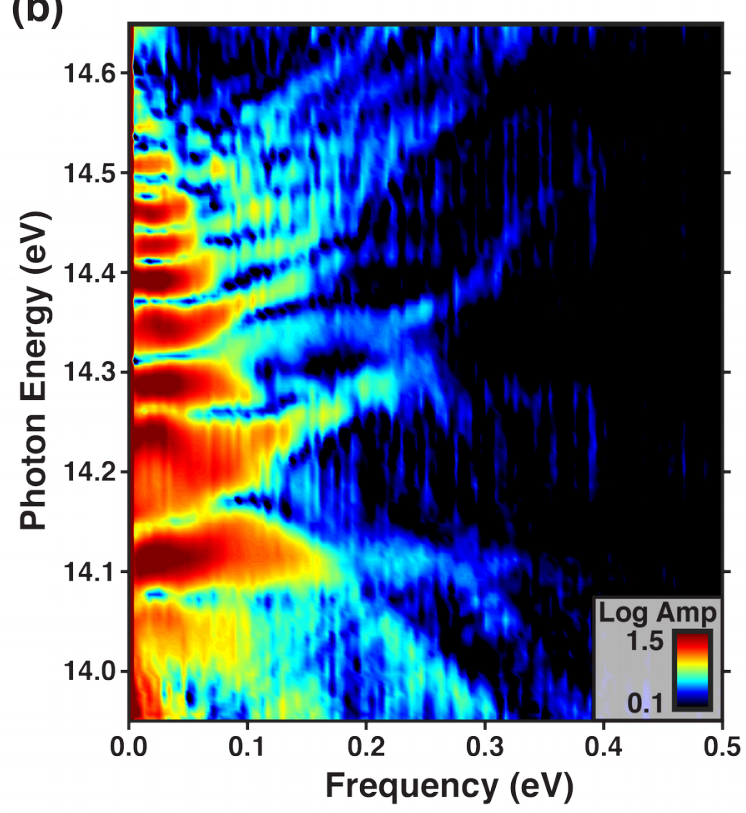

Supplementary Fig. 2: Transient absorption measurement of $\mathrm{Kr}$ autoionizing states over nearly a picosecond of delays. (a) Absorption features as a function of photon energy and of XUV-NIR delay. (b) Fourier transform of the delay-dependent spectrum. 


\section{Supplementary Note 3: Definition of the third order response function and perturbative simulations}

The broadband XUV that extends from 13.5 to $16.0 \mathrm{eV}$ generates a coherent superposition between the ground state and the excited states both above and below the first ionization potential at $14.0 \mathrm{eV}$. The response of the medium to this broadband coherent excitation can be described by a macroscopic polarization:

$$
P=\varepsilon_{0}\left(\chi^{(1)} E+\chi^{(2)} E E+\chi^{(3)} E E E+\ldots\right)
$$

where $\varepsilon_{0}$ is the vacuum permittivity, $\chi^{(n)}$ is the $n$-order susceptibility, and $E$ is the electric field of the excitation. With XUV pulse energies on the order of $1 \mathrm{~nJ}$, higher order terms can be neglected for the initial excitation. The first order polarization generated in the excited cross section of atoms emits a coherent field out of phase with the incident XUV light, resulting in depletion features at excited state energies. The temporal characteristics of this polarizationinduced emission, also called the free induction decay (FID), map to the coherence time of the excited state. ${ }^{1}$ After the initial XUV excitation, two time-coincident 6 fs NIR pulses probe the coherence as a function of XUV-NIR delay. The coherent interaction of the XUV-induced FID and these intense NIR pulses generates a macroscopic polarization with significant contributions from higher order terms. In an isotropic medium, these nonlinear terms result in emission from wave-mixing processes dependent upon odd orders of the nonlinear susceptibility, $x^{(n)}$.

In the time-domain, an $n$-th order polarization, $\quad P^{(n)}(t)$, can be described as the convolution of the electric fields and the nonlinear response function, $R^{(n)}\left(t_{n}, \ldots t_{1}\right)$ :

$$
P^{(n)}(t) \propto \int_{0}^{\infty} d t_{n} \int_{0}^{\infty} d t_{n-1} \ldots \int_{0}^{\infty} d t_{1} E\left(t-t_{n}\right) E\left(t-t_{n}-t_{n-1}\right) \cdot \ldots \cdot E\left(t-t_{n}-t_{n-1}-\ldots-t_{1}\right) \cdot R^{(n)}\left(t_{n}, \ldots t_{1}\right)
$$

where the $t$ variables denote time intervals defined by the pulse sequence. For the third order polarization, the response function may be expressed:

$$
R^{(3)}\left(t_{3}, t_{2}, t_{1}\right) \propto i\left\langle\mu\left(t_{3}+t_{2}+t_{1}\right)\left[\mu\left(t_{2}+t_{1}\right),\left[\mu\left(t_{1}\right),[\mu(0), \rho(-\infty)]\right]\right]\right\rangle
$$

where $\mu$ is the dipole operator and $\rho$ is the density matrix in the ground state. The response function can be expanded to yield eight terms, each of which describe a sequence of interactions with the density matrix. ${ }^{2}$ In the experimental configuration described here, where detection of the XUV-initiated dynamics occurs in the XUV, only two terms contribute to the observed time-dependent signals:

$$
R^{(3)}\left(t_{3}, t_{2}, t_{1}\right) \propto i\left\langle\mu\left(t_{3}+t_{2}+t_{1}\right) \mu\left(t_{2}+t_{1}\right) \mu\left(t_{1}\right) \mu(0) \rho(-\infty)\right)-i\left\langle\rho(-\infty) \mu(0) \mu\left(t_{1}\right) \mu\left(t_{2}+t_{1}\right) \mu\left(t_{3}+t_{2}+t_{1}\right)\right\rangle
$$

where $t_{2}=0$ to reflect simultaneous interaction of both NIR pulses. The pulse sequence as well as the generalized double-sided Feynman diagrams for these terms are provided in Supplementary Fig. 3a-b. Because the interactions occur on only one side of the diagram, these experiments yield the polarization, or dephasing, time, which is equal to twice the excited state lifetime.

The broad bandwidth pulses utilized in these experiments allow for the simultaneous excitation and NIR-mediated coupling of myriad one-photon, dipole-allowed (bright) states, leading to multiple Feynman diagrams with distinct initial interactions that emit at the same photon energy. 
The interferences of these different pathways result in quantum beat oscillations in the wavemixing signal with frequencies that correspond to energy differences between coupled bright

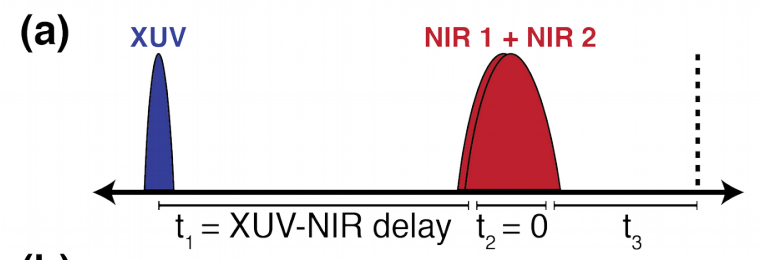

(b)

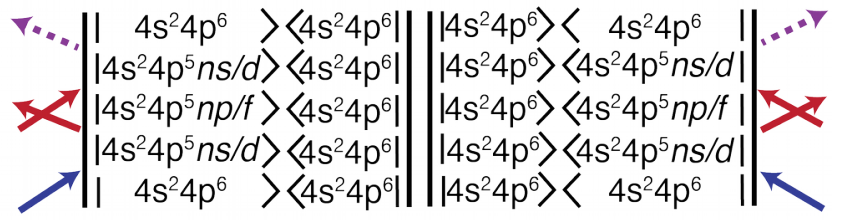

\section{Supplementary Fig. 3: Pulse sequence and generalized double-sided Feynman diagram for the described experiments. (a) Pulse sequence with times $t_{1}, t_{2}$, and $t_{3}$ defined. (b) Because dynamics are initiated and detected with the weak XUV pulse, only coherences are measured in this experiment.}

states, as observed in the experimental date. In a system that consists of states that decay at different rates, quantum beating indicates that the observed time evolution does not arise from the dynamics of a single state, but rather from the contributions of multiple different states.

The third order polarization resulting from the experimental geometry described above is simulated as a function of photon energy and pulse delay, $t_{1}$, for the $4 \mathrm{~s}^{2} 4 \mathrm{p}^{5}\left({ }^{2} \mathrm{P}_{3 / 2}\right) n l$ and $\left({ }^{2} \mathrm{P}_{1 / 2}\right)$ nl excited states of $\mathrm{Kr}$. A false color plot showing the results of these calculations is provided as Fig. 4a. The simulation incorporates 27 ns/nd bright states below the first IP (13.6 $-13.96 \mathrm{eV})$ and 26 bright states above the IP (14.07-14.63 eV) as well as $16 \mathrm{np} / \mathrm{nf}$ one photon dipole forbidden (dark) states accessible by NIR-mediated transitions from the autoionizing states above the first IP. All states were identified using atomic transition line data available from NIST. The lifetimes and photoabsorption cross sections of the lowest lying $\left({ }^{2} \mathrm{P}_{1 / 2}\right) n d / n s$ autoionizing states were obtained from literature lineshape measurements. ${ }^{3}$ For higher lying states for which no literature values could be found, approximate lifetimes were estimated by assuming that the radiative lifetime scales proportionally with $n^{3}$. The states below the first IP decay on timescales significantly longer than those investigated in these experiments. ${ }^{5,6}$ To better replicate experimental conditions, the transition dipoles were modulated by the measured XUV and NIR spectra. Only fully-resonant wave-mixing pathways in which the NIR photon energy is equivalent to the transition frequency between a bright state and one of the 16 dark states are considered. Long-lived oscillatory features appear at the energies of the $\left({ }^{2} \mathrm{P}_{1 / 2}\right) n d / n s$ autoionizing states. As demonstrated above, these oscillations arise from the interference of multiple different wave-mixing pathways with the same final state. The Fourier analysis (Supplementary Fig. 4b) reveals the frequency components of the complex oscillatory structures observed in the time domain. Each peak corresponds to the energy difference between two bright states participating in the XUV-prepared coherence. Because of the broad bandwidth of both the initial XUV pulse and the subsequent NIR pulses, many members of the coherence contribute to 
emission signals from states within the NIR bandwidth, leading to nearly identical beating patterns within all states.

These results stand in stark contrast to the experimentally measured spectrum in Fig. 3 of the main manuscript. While persistent quantum beat oscillations like those in Supplementary Fig. 4 are present at all delays in Fig. 3, a shorter-lived component nearly five times more intense than these quantum beats is also observed in the wave-mixing emission signals. The duration of the short-lived component of each wave-mixing emission signal corresponds to the lifetime of the $\left({ }^{2} \mathrm{P}_{1 / 2}\right)$ nd reporter state from which it emits. As explained in Section IVB, pathways employing what appears to be two degenerate NIR photons are enhanced relative to other pathways for the entire manifold of autoionizing states, allowing for the preferential coupling of the nd reporter states to themselves and the direct measurement of $n d$ state lifetimes. This result does not arise naturally from the perturbative simulation described above. Variations in bright to dark state transition dipole moments modify the relative contributions of different members of the coherence, but they should be reflected in all emitted signals. Furthermore, while multiple dark states participate in the wave-mixing process, they are not finely spaced enough to account for the observed trend in lifetimes or the variation in oscillatory frequencies, especially when approaching the second IP. Therefore, one plausible explanation for the NIR-degenerate pathway enhancement in the experimental data is nonperturbative effects, such as the AC-Stark effect, which has already been implicated in the delays observed between wave-mixing emission orders. More work is necessary to validate this possibility.

While similar NIR degenerate wave-mixing pathways coupling the longer-lived $\left({ }^{2} \mathrm{P}_{1 / 2}\right)$ ns states to themselves certainly contribute to the observed emission signals (see Fig. 8a for an example), the extent to which these pathways are enhanced is unclear. As shown in manuscript Fig. 3b, a Fourier transform taken over nearly a picosecond of XUV-NIR delays contains features that can be assigned NIR degenerate pathways coupling the $\left({ }^{2} \mathrm{P}_{1 / 2}\right) n s$ states, but lacks the pronounced unity slope lines observed in early-delay Fourier transform (Fig. 5b) attributed to enhancement of the NIR degenerate pathways that couple the $\left({ }^{2} \mathrm{P}_{1 / 2}\right)$ nd states. Degenerate pathways coupling the $\left({ }^{2} \mathrm{P}_{1 / 2}\right)$ ns states may contribute to the unity slope lines in the early-delay Fourier transform. However, these $\left({ }^{2} \mathrm{P}_{1 / 2}\right)$ ns pathways cannot be easily distinguished from the $\left({ }^{2} \mathrm{P}_{1 / 2}\right)$ nd pathways, prohibiting an assessment of the degree of enhancement, if any, of the NIR degenerate pathways coupling the $\left({ }^{2} \mathrm{P}_{1 / 2}\right) n s$ states. It is nonetheless important to note that the mechanism leading to the enhancement of wave-mixing pathways coupling the short-lived $\left({ }^{2} \mathrm{P}_{1 / 2}\right)$ nd states likely does not impact the longer-lived $\left({ }^{2} \mathrm{P}_{1 / 2}\right) n$ s states to the same degree. For example, previous literature has identified wave-mixing regimes in which nonperturbative effects become significant by examining the relationship between the Rabi frequency and the state's dephasing time. ${ }^{7,8}$ 
(a)

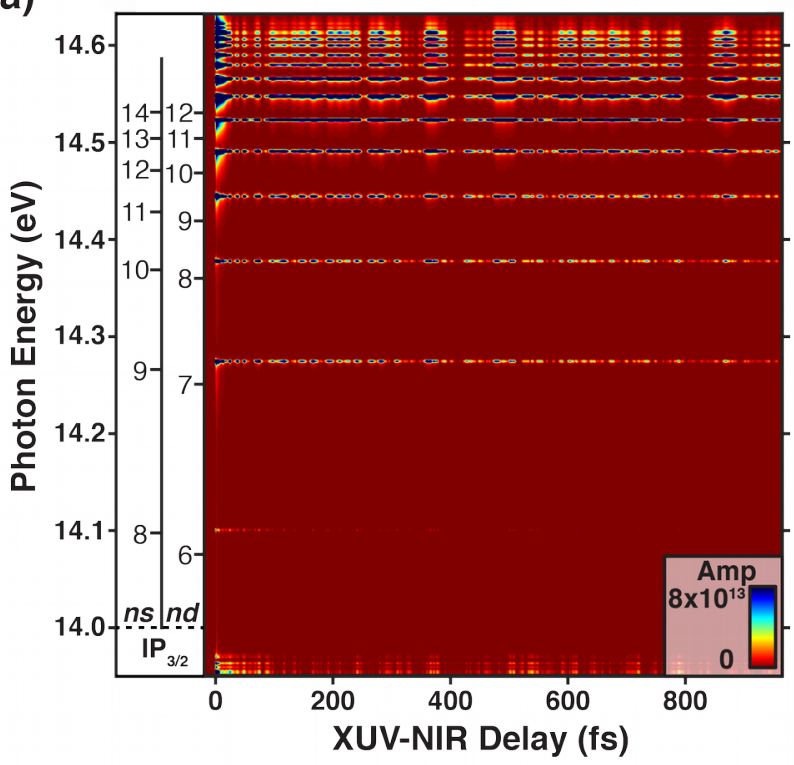

(b)

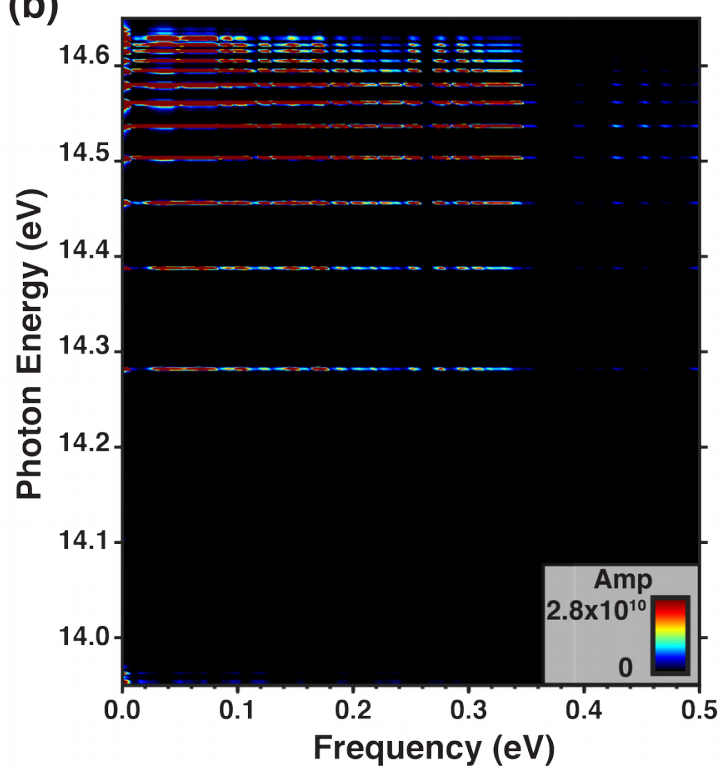

Supplementary Fig. 4: Perturbative four-wave-mixing simulations of autoionizing states of Kr. (a) A homodyne four-wave-mixing signal as a function of $t_{1}$, which is the XUV-NIR delay in our experiment, and of photon energy. (b) Fourier transform along $t_{1}$.

\section{References}

${ }^{1}$ S. Bengtsson, E.W. Larsen, D. Kroon, S. Camp, M. Miranda, C.L. Arnold, A. L'Huillier, K.J. Schafer, M.B. Gaarde, L. Rippe, and J. Mauritsson, Nat. Photonics 11, 252 (2017).

${ }^{2}$ P. Hamm and M.T. Zanni, Concepts and Methods of 2D Infrared Spectroscopy (Cambridge University Press, Cambridge, 2011).

${ }^{3}$ K. Maeda, K. Ueda, and K. Ito, J. Phys. B At. Mol. Opt. Phys. 26, 1541 (1993).

${ }^{4}$ W. Demtroder, Atoms, Molecules, and Photons (2015).

${ }^{5}$ P.G. Wilkinson, Can. J. Phys. 45, 1709 (1967).

${ }^{6}$ F.B. Dunning and R.F. Stebbings, Phys. Rev. A 9, 2378 (1974).

${ }^{7}$ J.C. Wright, Annu. Rev. Phys. Chem. 62, 209 (2011).

${ }^{8}$ N.A. Mathew, L.A. Yurs, S.B. Block, A. V. Pakoulev, K.M. Kornau, E.L. Sibert, and J.C. Wright, J. Phys. Chem. A 114, 817 (2010). 
